\title{
A semi-Lagrangian transport method for kinetic problems with application to dense-to-dilute polydisperse reacting spray flows
}

\author{
François Doisneau ${ }^{\mathrm{a}, *}$, Marco Arienti ${ }^{\mathrm{a}}$, Joseph C. Oefelein $^{\mathrm{a}}$ \\ ${ }^{a}$ Combustion Research Facility, Sandia National Laboratories, Livermore, CA 94551-0969, USA
}

\begin{abstract}
For sprays, as described by a kinetic disperse phase model strongly coupled to the Navier-Stokes equations, the resolution strategy is constrained by accuracy objectives, robustness needs, and the computing architecture. In order to leverage the good properties of the Eulerian formalism, we introduce a deterministic particle-based numerical method to solve transport in physical space, which is simple to adapt to the many types of closures and moment systems. The method is inspired by the semi-Lagrangian schemes, developed for Gas Dynamics. We show how semi-Lagrangian formulations are relevant for a disperse phase far from equilibrium and where the particle-particle coupling barely influences the transport; i.e., when particle pressure is negligible. The particle behavior is indeed close to free streaming. The new method uses the assumption of parcel transport and avoids to compute fluxes and their limiters, which makes it robust. It is a deterministic resolution method so that it does not require efforts on statistical convergence, noise control, or post-processing. All couplings are done among data under the form of Eulerian fields, which allows one to use efficient algorithms and to anticipate the computational load. This makes the method both accurate and efficient in the context of parallel computing. After a complete verification of the new transport method on various academic test cases, we demonstrate the overall strategy's ability to solve a strongly-coupled liquid jet with fine spatial resolution and we apply it to the case of high-fidelity Large Eddy Simulation of a dense spray flow. A fuel spray is simulated after atomization at Diesel engine combustion chamber conditions. The large, parallel, strongly coupled computation proves the efficiency of the method for dense, polydisperse, reacting spray flows.
\end{abstract}

Keywords: Kinetic theory, Fuel injection, Dense polydisperse reacting spray, Euler-Euler spray model, Semi-Lagrangian transport scheme, Two-way coupling.

\section{Introduction}

Kinetic equations describe systems of particles in a statistical way and as point particles. They can be used to model problems with a large number of particles. Such problems are ubiquitous in physics and mechanical engineering; e.g., plasmas, rarefied gases, stellar clouds, neutron beams, spray clouds, particle suspensions, and granular gases. Kinetic equations are intrinsically related to fluid descriptions $[45,6]$, but are generally used to account for systems that are far from equilibrium. They can be strongly coupled to other systems. For instance the Vlasov equation for charged particles [69] and the Williams-Boltzmann equation for polydisperse drops [95] are coupled to Maxwell and NavierStokes systems respectively and this coupling can be two-way. Such couplings actually constrain the choice of the resolution method, which is therefore case-dependent.

We are particularly interested in predicting spray injection, atomization, and combustion for mechanical engineering problems. In piston engines, adjusting the injection sequence is considered a viable way to improve efficiency while reducing pollutants. However, the injection process is multiphysics and multiscale: it involves the formation, transport, and evaporation of fuel drops that are $10^{3}$ smaller than the combustion chamber length. Despite the increase in computational power, the

\footnotetext{
${ }^{*}$ Corresponding author. Tel.: +1 (925) 2946738.

Email addresses: fdoisne@sandia.gov (François Doisneau), marient@sandia.gov (Marco Arienti), oefelei@sandia.gov (Joseph C. Oefelein)
} 
representation of all these scales, together with the physics of compressibility, turbulence, entrainment, atomization, spray transport, phase change, mixing, and turbulent combustion, still involves many challenges. The multiscale nature of the disperse phase can be partially mitigated by a kinetic description, provided that the numerical strategy is accurate simultaneously on the transport in physical space, on the evolution of the numerous degrees of freedom of the drop phase space, and on the strong couplings to the compressible, turbulent, and reacting gas phase. In particular the near-nozzle region, which hosts the onset of turbulent and atomizing phenomena that are crucial to the physics of spray penetration and mixing, is characterized by high mass loadings and volume fractions of liquid. This situation is hereafter referred to as a dense spray, that connects to the dilute spray where the stoichiometry allows combustion. It requires one to capture the strong, two-way coupling $[4,39]$ interactions in terms of mass, momentum, and heat transfers between the liquid and the gas. And for high volume fractions, the flow dynamics may also depend on particle-particle interactions (collisions, coalescence, and break-up), a situation sometimes referred to as four-way coupling [4]. Hence a comprehensive simulation strategy for direct fuel injection requires an accurate transport scheme for the kinetic description of sprays that can efficiently render strong coupling, including in the near nozzle region.

A widespread technique to solve kinetic equations is the stochastic Lagrangian approach. The qualification of stochastic is sometimes omitted but it is worth stressing the differences compared to deterministic Lagrangian approaches. The latter treatment would only be possible if the number of particles were sufficiently low and their initial and boundary conditions (IC/BC) sufficiently well known. On the contrary, the stochastic Lagrangian approach is insensitive to the physical number of particles and deals with IC/BC through modeled distributions. In most applications, the Lagrangian treatment of particles is in fact the result of a Monte-Carlo technique [40], so that it is referred to as Direct Simulation Monte-Carlo or DSMC by Bird [11]. DSMC computations therefore use parcels, which are misleadingly thought as being (bundles of) the physical particles. Still, many of the DSMC closures resemble the microscopic laws for a single particle, which makes DSMC a convenient choice together with its ease in describing high degrees of freedom of the underlying kinetic approach. It is an accurate way to describe the convection of particle clouds with multiple spatial scales and strong density gradients. Lagrangian methods have been widely applied to the simulation of sprays stemming from fuel injection $[40,75,1,2]$ and to particle-laden flows [28, 57, 66, 67]. Lagrangian methods are also applied to the simulation of charged particles in plasmas, the reader being referred to Birdsall and Langdon's monograph [12]. In the case of particle-laden flows, DSMC is considered as a reference method to solve the transport operator and is used to validate velocity closures [29, 72, 91]. It is also a well established approach in mechanical engineering problems such as gas turbines [87, 60], solar concentrators [96], and chemical engineering risers [17]. For internal combustion engines with direct fuel injection, DSMC descriptions of the spray have allowed Reynolds Average NavierStokes (RANS) simulations with multiple physical phenomena like a moving piston [90] or a detailed turbulent-chemistry interaction model [77] as well as Large Eddy Simulations (LES) [58, 93]. But stochastic Lagrangian simulations are difficult to converge leading to statistical noise and to spurious correlation patterns. For example in solid rocket boosters (SRB), the effect of aluminum oxide drop coalescence on polydispersity is sought in a space and time resolved fashion. But the dynamics of the aluminum oxide spray size distribution is rich enough for coalescence terms to require many DSMC samples to be present at a given location, typically more than 200 [57]. So that Lagrangian methods provide very noisy SRB results, even when coarse meshes are used in order to involve more samples in the local averages [37]. In general, the number of parcels with respect to physical space (particle size phase space) has a similar effect as the discretization of the physical (phase) space for Eulerian approaches. The uniformity of the parcels in spanning these spaces drives the accuracy of DSMC. But the uniformity strongly varies with time. The initial Monte-Carlo sampling may also influence the level of uniformity at each time in a way that depends on the flow. Therefore the evolution of sampling, which governs the accuracy of DSMC, is difficult to predict. Thus, efficiently converging DSMC simulations requires in fact knowledge of the conditionings and correlations stemming from the 
physics that is sought. Also, stochastic Lagrangian codes are more difficult to parallelize when two-way coupled to a phase described with the Eulerian formalism [50]. The coupling of two phases, one being described by a Lagrangian approach and the other one by an Eulerian approach, is sometimes referred to as the particle-in-cell method [24]. First, balancing the load among processors in the context of domain decomposition is made difficult. Stochastic Lagrangian parcels can indeed accumulate in some physical locations, whereas the Eulerian phase always has an even load by construction of the domains. Second, enforcing strong coupling between two phases which space discretizations do not match each other imposes a complex treatment $[62,78]$; e.g., a computationally extensive coupling between all the physical locations of the domain or an iterative procedure, resulting in a trade-off between accuracy and parallel efficiency.

As an alternative, one can consider Eulerian methods to solve kinetic equations; e.g., if any strong coupling exists (collisions, exchanges with another phase). The dynamics of charged particles in a plasma has been described on fixed grids using a Eulerian set of equations by many authors [20, 47, 46, 18]. However, Eulerian methods require the reduction of the phase space size to be tractable. For instance, the peculiar dynamics of Water-Bag models for plasmas stems from such assumptions [10], which are case-specific. Additional modeling based on assuming some conditioning between phase space variables brings in new systems of equations to approximate the original kinetic equation. In the case of the modeling and simulation of spray flames [29] and of aluminum particles in solid rocket motors [39], various assumptions can be made on the velocities of the particles such as the monokinetic assumption [29] or Gaussian closures [91]. They allow the reduction of the kinetic equation into a fluid system based on some moments. Devising such a system of fluid equations is the essence of Eulerian methods. However, the spatial waves in the macroscopic system (arising from microscopic agitation induced by particle collisions or turbulence) are slower and weaker than in gas dynamics (where they arise from molecule collisions) so that "fluids" of particles are highly compressible [33]. In general, each set of assumptions used to reduce the size of a kinetic problem will require a dedicated transport method. While most of the past 60 years' efforts on hyperbolic systems [51, 89, 82, 21] were oriented towards gas dynamics (incompressible, low Mach, supersonic, hypersonic), the resulting schemes are not suitable for disperse phase flows. In particular, dedicated numerical methods are required to treat such hypercompressible transport in physical space [29, 63, 92, 81]. Still Eulerian methods have been applied with success to mechanical engineering problems, especially when the coupling is strong (through two-way momentum and heat transfers or through evaporation and combustion). Examples of applications are gas turbine ignition [13], Diesel spray polydisperse evaporation [42, 61] and combustion LES [88], SRB hydroacoustic [39] and thermoacoustic [83] instabilities, and fluidized beds [44]. Eulerian methods have been acknowledged for their good coupling and parallel properties in the spray $[13,37]$ and plasma communities [52].

For a kinetic equation with a strong coupling, it appears profitable to perform the transport with a method that has Lagrangian features, while keeping the data in the form of a deterministic Eulerian field. Computing the time evolution of hyperbolic equations by integrating along characteristics of the system with some projection to the Eulerian grid is referred to as a semi-Lagrangian technique in the literature. It has been introduced by [25] with a forward (explicit) and a backward (implicit) version, the latter being widely used in geosciences for global computations [94, 64, 80, 86, 84] and also in plasma dynamics $[30,20,85,52]$. Advection dominated problems, such as passive scalar transport, are good candidates for forward semi-Lagrangian approaches; e.g., methods have been recently proposed for weather or climate forecasts $[22,14]$. In the context of a disperse phase of droplets or particles, semi-Lagrangian methods are attractive because they are naturally compatible with the weakly-coupled nature of the material elements that are transported; i.e., weak diffusion and collisions, and slow or zero-velocity waves allow the characteristics to remain fairly independent of each other. An attempt for a one-way coupled spray has been made using backward semi-Lagrangian [76] and a more recent emphasis has been put on achieving non-oscillatory high-order schemes for dilute weakly collisional sprays [7]. The connection between semi-Lagrangian schemes and the remeshing process for stochastic Lagrangian methods has been established [23], emphasizing the conditions to 
achieve conservation properties. The issues that have been tackled in these works become even more significant when strong coupling occurs because additional constraints on regularity and oscillations have to be enforced. In plasma physics, a forward formulation has been devised $[73,26]$ to solve the Vlasov-Maxwell system, which is a strongly coupled fluid-kinetic model for the description of charged particles in their own mean field. The similarities of this system with that of dense, two-way coupled sprays are strong except that the equation of the coupled phase is elliptic (Maxwell) in the first case and hyperbolic (Navier-Stokes) in the second case. So semi-Lagrangian approaches are a viable strategy to perform the transport of kinetic and kinetically derived systems (with internally weak coupling) and they are promising for strongly coupled multiphysics problems such as dense sprays if the strong coupling (to an external phase) can be properly achieved.

In this paper, a forward semi-Lagrangian transport scheme is introduced, validated, and discussed as an efficient way to eliminate stochastic noise. The motivation for a semi-Lagrangian scheme is also presented. Such a method can naturally enforce conservativity and realizability as well as monotonicity. It is simple to implement compared to the complexity of computing Eulerian fluxes, and allows the use of off-the-shelf particle detection algorithms. The method is developed explicitly for use with the Large Eddy Simulation (LES) technique in the context of atomization and spray injection [35]. It is one of the features of the present work to extend the use of disperse spray models to the more dense, near-nozzle region, benefitting from the increased robustness of the developed method. Recognizing the strong density differences between the high-speed liquid and the high-pressure chamber gas, it is crucial for the numerical method to be able to robustly describe the steep gradients between regions dominated by liquid and by gas as well as the multiple scales that arise in detailed computations.

The paper is organized as follows. Section 2 describes the forward semi-Lagrangian (FSL) principles for the pure transport of a kinetic equation. We discuss the properties of the chosen projection formula, a linear projection leading to the definition of the FSL-LP transport scheme. Section 3 describes a strongly coupled polydisperse spray model based on kinetic theory and sectional discretization of droplet sizes. A full resolution strategy is introduced based on a tailored operator splitting and the FSL scheme. We give details on the strategy and its implementation in a high-fidelity LES solver, the RAPTOR code, developed by Oefelein [74], in Section 4, together with a verification of the coupling solver on two-way-coupling. Verification cases for the FSL-LP transport scheme are then presented with increasing complexity in Section 5. They show key properties of the approach when used for complex simulations of sprays, as compared to DSMC. We then verify and discuss the spray strategy for dense sprays in the near-nozzle region in Section 6 by comparison to a reference solver providing an interface capturing DNS of the early liquid jet. Finally in Section 7, the new strategy is applied to Diesel spray problems with a realistic computational size using RAPTOR. In particular, reacting polydisperse results demonstrate the strategy's feasibility and good scalability, and therefore its relevance to massively parallel simulations. We conclude on the new strategy and its prospects for spray simulations.

\section{General description of the transport method}

A forward semi-Lagrangian transport scheme is introduced for the pressureless gas dynamics equations, as they are the simplest Eulerian moment system describing free streaming of particles.

\subsection{Base model}

We consider a simple kinetic equation describing the free streaming of particles. Particles are accounted for using a number density function $f(t, \boldsymbol{x} ; \boldsymbol{c})$ or NDF:

$$
\partial_{t} f+\partial_{\boldsymbol{x}} \cdot(\boldsymbol{c} f)=0
$$

with $t$ the time, $\boldsymbol{x}$ the position in the 3D physical space, and $\boldsymbol{c}$ the particles' internal coordinate spanning the velocity phase space, therefore of dimension 3. On one hand, Eq. (1) can be solved using 
a stochastic Lagrangian approach [66] or Direct Simulation Monte-Carlo (DSMC) by approximating the NDF as composed of samples $s$ or parcels

$$
f(t, \boldsymbol{x} ; \boldsymbol{c}) \approx \sum_{s=1}^{N_{\text {samples }}} w_{s}(t) \delta\left(\boldsymbol{x}-\boldsymbol{x}_{s}(t)\right) \delta\left(\boldsymbol{c}-\boldsymbol{u}_{s}(t)\right),
$$

which results in the following $N_{\text {samples }}$ systems of ODEs

$$
\left\{\begin{array}{l}
\dot{w}_{s}(t)=0 \\
\dot{\boldsymbol{x}}_{s}(t)=\boldsymbol{u}_{s}(t) \quad, \quad s \in \llbracket 1, N_{\text {samples }} \rrbracket \\
\dot{\boldsymbol{u}}_{s}(t)=0
\end{array}\right.
$$

where the equation for $\boldsymbol{x}_{s}$ is similar to an equation for the characteristic curves. Good accuracy is reached if index $s$ spans parcels with varied enough positions and velocities, therefore requiring $N_{\text {samples }}$ to be large. As a stochastic method, the error decreases with $1 / \sqrt{N_{\text {samples }}}$ if no biasing is introduced in the sampling initialization process. On the other hand, Eq. (1) is equivalent to the following moment system for monokinetic initial conditions and in the absence of trajectory crossing [16, 29]:

$$
\left\{\begin{array}{l}
\partial_{t} \rho+\partial_{\boldsymbol{x}} \cdot(\rho \boldsymbol{u})=0 \\
\partial_{t} \rho \boldsymbol{u}+\partial_{\boldsymbol{x}} \cdot(\rho \boldsymbol{u} \otimes \boldsymbol{u})=0
\end{array} \quad \text { where } \quad\left(\begin{array}{c}
\rho \\
\rho \boldsymbol{u}
\end{array}\right)(t, \boldsymbol{x})=\int_{\boldsymbol{c} \in \mathbb{R}^{3}}\left(\begin{array}{l}
1 \\
\boldsymbol{c}
\end{array}\right) f(t, \boldsymbol{x} ; \boldsymbol{c}) \mathrm{d} \boldsymbol{c} .\right.
$$

This Eulerian system describes the so-called pressureless gas dynamics (PGD) $[97,15]$ and requires the use of specific numerical schemes $[16,29,81]$ that are at best second order space accurate. Despite the numerical peculiarities arising from Eq. (3), this representation is preferred to DSMC in some cases. The motivations for a Eulerian model will appear in the following.

\subsection{Forward Semi-Lagrangian scheme: a gather-scatter sequence}

We introduce an algorithm that deterministically solves the Eulerian fields of Eq. (3), that avoids flux computations, and instead resorts to the transport of parcels as in DSMC. We refer to it as Forward Semi-Lagrangian (FSL). Since we are solving a pressureless system, that is a fluid model for decoupled particles, the scheme is equivalent to a simple gather-scatter sequence with trivial transport. The approach is as follows. We consider a finite volume mesh; i.e., a partition of the physical space, with $N_{\text {cells }}$ cell centers $\boldsymbol{x}_{i}$ and cell volumes $\mathcal{V}_{\text {cell }}\left(\boldsymbol{x}_{i}\right)$.

1a) Scatter: Parcels are defined from Eulerian fields at time $t_{0}$ with their positions that match the cell centers. At a given node, as many parcels can be defined as needed to describe the Eulerian information with the desired accuracy: multiple (but discrete) velocities to render crossings or dispersion, multiple (discrete or continuous) sizes, temperatures, shapes etc. For the present PGD case, one parcel per cell is sufficient so that $N_{p}=N_{\text {cells. }}$. The mapping from an Eulerian to a Lagrangian (mesh-less) description at time $t$ is then given by:

$$
\left(\begin{array}{c}
\rho \\
\rho \boldsymbol{u}
\end{array}\right)\left(t_{0}, \boldsymbol{x}_{i}\right) \text { with } i \in \llbracket 1 ; N_{\text {cells }} \rrbracket \rightarrow\left(\begin{array}{c}
\boldsymbol{x}_{p} \\
m_{p} \\
\boldsymbol{u}_{p}
\end{array}\right)\left(t_{0}\right) \text { with } p \in \llbracket 1 ; N_{p} \rrbracket,
$$

where $\boldsymbol{x}_{p}\left(t_{0}\right)=\boldsymbol{x}_{i}, m_{p}\left(t_{0}\right)=\rho\left(t_{0}, \boldsymbol{x}_{i}\right) \mathcal{V}_{\text {cell }}\left(\boldsymbol{x}_{i}\right)$, and $\boldsymbol{u}_{p}\left(t_{0}\right)=\boldsymbol{u}\left(t_{0}, \boldsymbol{x}_{i}\right)$.

1b) Transport: All of the Lagrangian parcels are advected at their own velocity over $\Delta t$. The characteristics of Eq. (3) are straight lines, as it appears from Eq. (2). So that this step is a trivial linear extrapolation.

2a) Gather: At $t_{0}+\Delta t$, the Lagrangian information is now at arbitrary locations. A projection (devised below) is used to gather the Lagrangian information at the fixed, Eulerian cell centers. This projection is linear in $\rho$ but will induce an overall non-linearity in the case where velocities are averaged at the cell centers. At this point, the Eulerian field is the approximated solution of Eq. (3) at time $t_{0}+\Delta t$. 
This ends the transport cycle over a time step $\Delta t$. As a remark, a link can be established between the weights $w_{s}(t)$ from DSMC and the weights $m_{p}(t)$ from FSL. For parcels $s$ from the DSMC method lying in the vicinity of a cell $\boldsymbol{x}_{i}$, the $w_{s}(t)$ weights should sum up to the $m_{p}(t)$ weights emitted from $\boldsymbol{x}_{i}$. A similar argument applies for momentum. This notion of local conservation may not strictly be retrieved in practice because the two methods introduce their own numerical errors, with sampling noise for DSMC and numerical diffusion for FSL. Still the two methods are strictly conservative at the global scale: we have on the whole mesh and at all times $\sum_{s=1}^{N_{\text {samples }}} w_{s}(t)=\sum_{p=1}^{N_{p}} m_{p}(t)$ for mass and $\sum_{s=1}^{N_{\text {samples }}} w_{s}(t) \boldsymbol{u}_{s}(t)=\sum_{p=1}^{N_{p}} m_{p}(t) \boldsymbol{u}_{p}(t)$ for momentum.

\subsection{Linear projection}

A key attribute of our formulation is its ability to transform an instantaneous Lagrangian description (point-wise masses at arbitrary locations after step 2a) into an Eulerian description (fixed locations). To accomplish this, a projection operation is needed, which is described next.

A conservative projection is performed on the $2^{n_{\text {dim }}}$ closest neighbors, where $n_{\text {dim }}$ is the dimension of the physical space. It only needs the knowledge of the parcel's and cell centers' positions and is performed independently for each parcel. The weights are based on the Cartesian-projected distances of the parcel to the cell centers, as shown in Fig. 1. An Eulerian node $\boldsymbol{x}_{i}=\left(x_{i}, y_{i}, z_{i}\right)$ receives mass from parcel $p$ under the condition

$$
p \in \Omega_{\boldsymbol{x}_{i}}=\left\{p \mid \boldsymbol{x}_{i}-\left(\begin{array}{c}
\Delta x^{-} \\
\Delta y^{-} \\
\Delta z^{-}
\end{array}\right)<\boldsymbol{x}_{p}<\boldsymbol{x}_{i}+\left(\begin{array}{c}
\Delta x^{+} \\
\Delta y^{+} \\
\Delta z^{+}
\end{array}\right)\right\},
$$

where $\Delta x^{ \pm}=\left|x_{i}-x_{i \pm 1}\right|$ so that parcels in immediately neighboring cells contribute to a cell as long as they are not located beyond the center of the neighbors. The repartition is done using a weight function $w_{p i}$, which is defined to have the following properties.

- Isotropy: The weight $w_{p i}$ is direction-independent so 1 D-weights $w_{p i}=\prod_{\alpha \in x, y, z} w_{p i, \alpha}$ can be
defined. - Linearity: The $1 \mathrm{D}$-weights $w_{p i, x}$ depend only and linearly on the normalized distance to the cell center $\eta_{x}=\frac{x_{p}-x_{i}}{x_{i \pm 1}-x_{i}}$.

- Convexity: For particles in cell $x_{i}$, the nearest neighbor $x_{i \pm 1}$ guarantees that $\eta_{x} \in[0,1]$.

- Consistency: The mass goes completely to $x_{i}$ if $x_{p}$ matches $x_{i}$ so that $w_{p i, x}=1$ if $\eta_{x}=0$.

- Conservativity: The weight is symmetric; i.e., $w_{p i, x}\left(1-\eta_{x}\right)=1-w_{p i, x}\left(\eta_{x}\right)$.

A particular property resulting from the last criterion is that the mass fully goes to $x_{i \pm 1}$ if $x_{p}$ matches $x_{i \pm 1}$ so that $w_{p i, x}=1$ if $\eta_{x}=0$. We finally choose the normalized distance to the neighboring cells' centers, which is the only linear choice,

$$
w_{p i, x}=1-\eta_{x}, \quad w_{p i, y}=1-\eta_{y}, \quad w_{p i, z}=1-\eta_{z}
$$

where the definition of $\eta_{x}$ is based on wether $\boldsymbol{x}_{p}$ is closer to $x_{i-1}$ or $x_{i+1}$ as said previously (convexity). Mass conservation is then enforced as the seven (three or one) other neighbors of $p$ in 3D (in 2D or in 1D) will receive contributions that are weighted by all of the possible products with the coefficients $w_{p i \pm 1, x}=1-w_{p i, x}, w_{p i \pm 1, y}=1-w_{p i, y}$, and $w_{p i \pm 1, z}=1-w_{p i, z}$. These are complementary to one thanks to the symmetry property. After gathering the old parcels within $\Omega_{\boldsymbol{x}_{i}}$, we get new values for parcels which positions now match the cell centers ("remeshing" point of view)

$$
\left(\begin{array}{c}
\rho_{i}=\sum_{p \in \Omega_{\boldsymbol{x}_{i}}}^{x_{i}} w_{p i} m_{p} \\
\rho_{i} \boldsymbol{u}_{i}=\sum_{\substack{p \in \Omega_{\boldsymbol{x}_{i}} \\
6}} w_{p i} m_{p} \boldsymbol{u}_{p}
\end{array}\right)
$$


and are readily used as the updated values for the Eulerian fields $\rho\left(t, \boldsymbol{x}_{i}\right)$ and $\boldsymbol{u}\left(t, \boldsymbol{x}_{i}\right)$ ("projection" point of view). The old, Lagrangian parcels are removed so that the spatial index is now $i \in \llbracket 1, N_{\text {cell }} \rrbracket$ while the old index $p \in \llbracket 1, N_{s} \rrbracket$ is no longer used. We now have exactly $N_{\text {cell }}$ parcels.

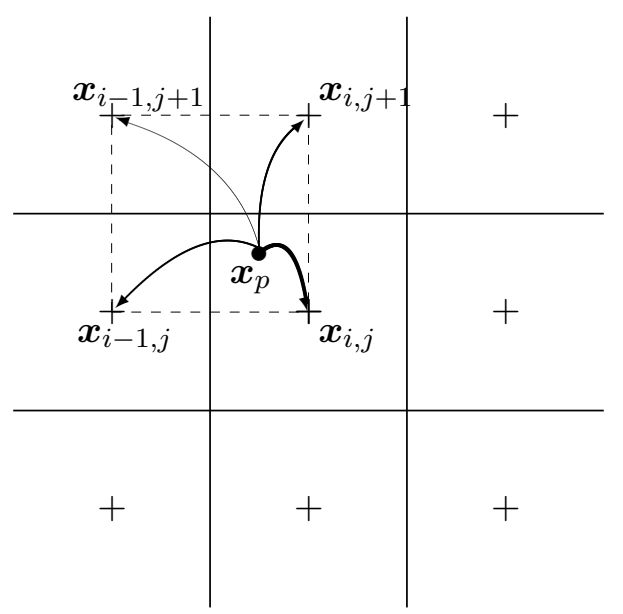

Figure 1: Schematic of the projection step for particle $p$ in a $2 \mathrm{D}$ structured mesh.

To summarize, the linear projection is algorithmically simple since it is performed independently for each particle and only requires knowledge of the mesh geometry. Most of its cost arises from the need to map each parcel to a cell of the mesh. This is performed once per cycle, which is as much as needed by DSMC to perform any kind of coupling to an Eulerian field. The linear projection is conservative to machine precision. Its order is limited because of the choice of linearity as shown in Appendix A. This projection has also been used in [73, 27]. It has been mathematically analyzed in 1D [79] and referred to as a linear B-spline by the same authors. From now on, we refer to the forward semi-Lagrangian scheme with linear projection as FSL-LP.

\subsection{Theoretical accuracy of the method}

The space-time order of semi-Lagrangian methods can be of the form $[9,79,43]$

$$
\mid \mathrm{SL} \text { error } \mid<C_{1} \Delta t^{p}+C_{2} \frac{\Delta x^{r+1}}{\Delta t} .
$$

In the specific case of a Verlet time integration, [79] prove the $L^{1}$ convergence of the method and an error $O\left(\Delta t^{2}+\Delta x^{2}+\frac{\Delta x^{2}}{\Delta t}\right)$. This gives a guidance on what can be expected in our case and for other time integration strategies.

The first part of the error is introduced by the characteristic reconstruction, which is exact in our case so that $C_{1}=0$. The second part is introduced by the projection scheme, which space accuracy is of order 2 in our case as demonstrated later, so that $r=1$. Thus, for a fixed time step, we expect for FSL-LP a rate of convergence of order 2 with the cell size. The characteristic which is enforced during the transport step is exactly that of Eq. (1), that is the initial kinetic equation; i.e., it describes free streaming. Conversely, this characteristic is not the one of the PGD problem of Eq. (3) as soon as a non-linearity (crossing) arises. If a crossing were to occur within a time step, the velocity averaging is not reproduced by the transport step of the semi-Lagrangian approach. The PGD treatment of trajectory crossings is recovered at the projection step; i.e., the particles with multivariate velocities are averaged into a "monokinetic" cloud. So resorting to large CFLs, though not a threat to stability per se, increases the risk of discrepancies to the exact PGD solution.

An analytical formula for the evolution of a Dirac $\delta$-function of density under successive projections is derived in Appendix A. This study confirms that the method introduces an error of order 2, which is diffusive and dependent on the CFL number. This study also illustrates the unconditional stability of the approach, allowing CFL numbers larger than 1. 
The method's order can be increased by considering higher order projections involving larger stencils $[73,27,23]$. The coefficients then stem from the choice of a projection kernel as extensively discussed in Cottet et al. [23]. These authors show the efficiency of a dimensional splitting of the projection, reducing the problem to choosing a proper 1D kernel based on compacity and smoothness arguments. The important question is then to cope with oscillatory behavior in under-resolved and discontinuous regions. Preserving realizability and in particular positivity is of paramount importance in our fully coupled spray context, while high-order demonstrations have been made for the transport of passive (one-way coupled) scalars [23]. The path is to perform a limitation of the reconstructed values [7] when local gradients are strong. Moreover in the case of moment methods, gradients of all the moments have to be examined; i.e., the projection should depend on the other particles' mass, momentum, and higher order moments (considered below). But this step has to be done in a conservative and positivity preserving fashion as conservation is another priority. In the present, we disregard devising higher order methods for the sake of conservativity and robustness but we keep in mind that the numerical diffusion of the linear projection might be impractical for some problems.

\section{Case of sprays}

\subsection{Fluid-kinetic model}

We consider a spray; i.e., disperse liquid structures suspended in a carrier gas. It is described with a fluid-kinetic model [95] using a set of continuous fluid equations for the gas (compressible NavierStokes system), which is strongly coupled to a kinetic equation for the disperse phase. The internal coordinates translate the degrees of freedom of particles, which are now droplets, and are the velocity vector, droplet temperature, and radius $(\boldsymbol{c}, \theta, r)$. Coupling is achieved through mass, momentum, and enthalpy source terms accounting for evaporation, drag, and heat transfer in a conservative way. This description is sufficient if the volume fraction of the disperse phase is not too high, and it can be easily closed if the liquid structures are spherical enough [66, 29]. The fluid-kinetic model is given by the system

$$
\left\{\begin{array}{l}
\partial_{t}\left(\rho_{g} Y_{l}\right)+\partial_{\boldsymbol{x}}\left(\rho_{g} Y_{l} \boldsymbol{u}_{g}\right)=-\partial_{\boldsymbol{x}} \mathbf{q}_{l}+\omega_{l}, \quad l \in \llbracket 1 ; N_{\text {species }} \rrbracket, l \neq f \\
\partial_{t}\left(\rho_{g} Y_{f}\right)+\partial_{\boldsymbol{x}}\left(\rho_{g} Y_{f} \boldsymbol{u}_{g}\right)=-\partial_{\boldsymbol{x}} \mathbf{q}_{f}+\omega_{f}+\int_{\boldsymbol{c}, \theta, r}(m(r) \mathrm{E}) f \mathrm{~d} \boldsymbol{c} \mathrm{d} \theta \mathrm{d} r \\
\partial_{t}\left(\rho_{g} \boldsymbol{u}_{g}\right)+\partial_{\boldsymbol{x}}\left(\rho_{g} \boldsymbol{u}_{g} \otimes \boldsymbol{u}_{g}\right)=-\partial_{\boldsymbol{x}} p-\partial_{\boldsymbol{x}} \mathbf{S}+\int_{\boldsymbol{c}, \theta, r}(-\mathbf{F}+\boldsymbol{c} \mathrm{E}) f \mathrm{~d} \boldsymbol{c} \mathrm{d} \theta \mathrm{d} r \\
\partial_{t}\left(\rho_{g} e_{g}\right)+\partial_{\boldsymbol{x}}\left(\rho_{g} e_{g} \boldsymbol{u}_{g}\right)=-p \partial_{\boldsymbol{x}} \boldsymbol{u}_{g}-\partial_{\boldsymbol{x}} \mathbf{q}+\int_{\boldsymbol{c}, \theta, r}\left(-\mathrm{H}-\mathbf{F} \cdot\left(\boldsymbol{u}_{g}-\boldsymbol{c}\right)+h(\theta, r) \mathrm{E}\right) f \mathrm{~d} \boldsymbol{c} \mathrm{d} \theta \mathrm{d} r \\
\partial_{t} f+\partial_{\boldsymbol{x}}(\boldsymbol{c} f)+\partial_{\boldsymbol{c}}(\mathbf{F} f)+\partial_{\theta}(\mathrm{H} f)+\partial_{r}(\mathrm{E} f)=\mathfrak{B}+\mathfrak{C},
\end{array}\right.
$$

where the indices $l$ refer to the chemical species together with index $f$ which refers to the one gas species composing the evaporation product from the liquid. The various source terms describe chemical reaction $\left(\omega_{f}\right.$ and all the $\left.\omega_{l}\right)$, evaporation $(\mathrm{E})$, drag $(\mathbf{F})$, heating $(\mathrm{H})$, break-up $(\mathfrak{B})$, and coalescence $(\mathfrak{C})$. The integrals defining the coupling terms in the Navier-Stokes equation are computed from the knowledge of drop closures $m(r)$ being the mass and $h(r, \theta)$ being the enthalpy. For the sake of legibility we have omitted use of filter notation associated with LES and we refer the reader to the literature for turbulent fluxes to be added in the species flux $\mathbf{q}_{l}$, stress tensor $\mathbf{S}$, and heat flux $\mathbf{q}$. As for the effect of filtering on the disperse phase equation, we do not account for it and leave this important research topic for later [34]. Any effect of the highly-loaded disperse phase on the filtered terms in the Navier-Stokes system [48] is left aside for the same reasons. We also neglect $\omega_{f}, \omega_{l}, \mathfrak{B}$, and $\mathfrak{C}$ in the present study since they do not contribute to gas-liquid coupling, keeping in mind that the present method can be directly extended to the treatment of these terms. Here we focus on evaporation, drag, and heating as they are inducing the coupling of interest between the two phases. 


\subsection{Semi-kinetic models}

The number density function (NDF) in kinetic equations always has a high dimensional phase space due to the velocity variable. In the above fluid-kinetic Eq. (6), the disperse phase NDF $f(t, \boldsymbol{x} ; \boldsymbol{c}, \theta, r)$ has $5 \mathrm{D}$ in addition to the $4 \mathrm{D}$ of space and time. For sprays, where the dynamics strongly depends on the droplet sizes, a reduction methodology relies on size-conditioning and shape-presuming [66]. After size conditioning, the shape-presuming reduction effort can be thought of two distinct modeling steps: i) the velocity treatment (on a size-conditioned basis) that leads to the various models for transport in physical space and ii) the size treatment.

Whatever kinetic equation, step i) is a classical approach. The reduction of dimensionality of the NDF through assumptions on velocity distributions leads to a wealth of Eulerian systems, so-called hydrodynamic models. In the spray case, the system with size-conditioned velocities is sometimes referred to as semi-kinetic because the size variable remains a continuous one, adding a dimension compared to usual hydrodynamic models [66]. Assuming perfectly correlated velocities; i.e. the monokinetic closure

$$
f(t, \boldsymbol{x} ; \boldsymbol{c}, \theta, r) \approx \sum_{k=1}^{N_{\mathrm{sec}}} \kappa_{k}(t, \boldsymbol{x} ; r) \delta\left(\boldsymbol{c}-\boldsymbol{u}_{k}(t, \boldsymbol{x})\right) \delta\left(\theta-T_{k}(t, \boldsymbol{x})\right),
$$

leads to a Eulerian system and is valid as long as $r$ is below a critical size, above which trajectory crossings will occur, in vortical and stretched regions of the flow. This closure can be seen as the zero-temperature limit of the Maxwell-Boltzmann distribution and leads to PGD for which the FSL scheme has been proposed in Section 2.1. PGD is relevant for particles/drops because the gas drag force tends to correlate the velocities locally in physical space, whereas other velocity closures may apply to other kinetic equations. For instance in the Vlasov equation for plasma physics, the selfconsistent collective electric field is strongly dependent on the local NDF, which invites other velocity closures. The so-called Water-Bag model is based on constant NDF values within bounded, simply connex, velocity domains [31] and has a different hydrodynamic structure and constitutive equation of state $[8,53]$. The plasma description can be extended to Multi-Water-Bag model by grouping the particles in overlapping velocity bins coupled through the electric field acting as a momentum source term $[31,10]$. For Water-Bag models, the pressure depends on the density gradients so the advectiondominated character, depending on initial conditions and time integration scales, should be quantified to devise the appropriate transport scheme. For sprays, the monokinetic assumption can been relaxed, leading to models accounting for statistical trajectory crossings; e.g., caused by turbulence [91, 19]. Yet for these closures, the pressure (tensor) that builds up in inertial areas due to crossings usually remains weak compared to advection so that FSL retains relevance for inertial drop transport models. For simplicity, we apply the monokinetic assumption, while seeking accuracy on the droplet size by discretizing the size phase space.

\subsection{Multi-Fluid model}

For the size treatment step ii), we consider a finite volume discretization referred to as Multi-Fluid, which is, in fact, an extension of sectional methods that can include sections with independent velocity treatments [66]. The entire size distribution can be accounted for by combined moment and MultiFluid methods. It can be efficiently transported with arbitrary accuracy, allowing one to render the missing global size moments; e.g., the Sauter Mean Diameter. The Multi-Fluid approach can uniquely reconstruct any NDF from the knowledge of a set of moments in size, velocity, and temperature,

$$
\left(\begin{array}{c}
m_{k, 0} \\
\cdots \\
m_{k, n} \\
m_{k, 3} \boldsymbol{u}_{k} \\
m_{k, 3} T_{k}
\end{array}\right)(t, \boldsymbol{x})=\int_{r_{k-1}}^{r_{k}} \int_{\mathbb{R}^{+}} \int_{\mathbb{R}^{3}}\left(\begin{array}{c}
1 \\
\cdots \\
r^{n} \\
r^{3} \boldsymbol{c} \\
r^{3} \theta
\end{array}\right) f(t, \boldsymbol{x} ; \boldsymbol{c}, \theta, r) \mathrm{d} \boldsymbol{c} \mathrm{d} \theta \mathrm{d} r, \quad k \in \llbracket 1 ; N_{\mathrm{sec}} \rrbracket
$$


where the number of moments $n$ is related to the number of unknown parameters in the shapes $\kappa_{k}$ that are presumed in each section $k$ [68] The equivalence above holds as long as $r_{0}<r_{1}<\ldots<r_{N_{\mathrm{sec}}}=+\infty$ is a partition of the size phase space. The $N_{\mathrm{sec}}$ intervals are referred to as the sections $[66,38]$. The Multi-Fluid modeling strategy has been applied to engine sprays [29] and specific extensions were considered [41, 61, 42] in cases where the numerical setup was limiting local drop concentrations to the dilute spray regime; e.g., by injecting liquid downstream of the nozzle. The strategy and numerics devised here focus on capturing near-nozzle scales and connecting dense spray dynamics to a dilute reacting spray. A review of spray injection and autoignition simulations [36] gives practical arguments to support this strategy.

Choosing two moments is typically a good trade-off [39] for polydisperse problems with diverse drop dynamics. In the following, we therefore resort to two moments in size per section. These are number and mass $\left(m_{k, 0}, m_{k, 3}\right)$, simply noted $\left(n_{k}, m_{k}\right)$, so we need to account for 6 scalar moments $\left(n_{k}, m_{k}, m_{k} \boldsymbol{u}_{k}, m_{k} T_{k}\right)$ per section. The disperse phase is therefore described by $6 N_{\text {sec }}$ scalar Eulerian fields in $4 \mathrm{D}$, instead of a scalar 9D problem. The final governing system of equations then reads

$$
\left\{\begin{array}{ll}
\partial_{t}\left(\rho_{g} Y_{l}\right)+\partial_{\boldsymbol{x}}\left(\rho_{g} Y_{l} \boldsymbol{u}_{g}\right) & =-\partial_{\boldsymbol{x}} \mathbf{q}_{l}, \quad l \in \llbracket 1 ; N_{\text {species }} \rrbracket, l \neq f \\
\partial_{t}\left(\rho_{g} Y_{f}\right)+\partial_{\boldsymbol{x}}\left(\rho_{g} Y_{f} \boldsymbol{u}_{g}\right) & =-\partial_{\boldsymbol{x}} \mathbf{q}_{f}+\sum_{k} \mathrm{E}_{k}^{m-g} \\
\partial_{t}\left(\rho_{g} \boldsymbol{u}_{g}\right)+\partial_{\boldsymbol{x}}\left(\rho_{g} \boldsymbol{u}_{g} \otimes \boldsymbol{u}_{g}\right) & =-\partial_{\boldsymbol{x}} p-\partial_{\boldsymbol{x}} \mathbf{S}+\sum_{k}\left(-\mathbf{F}_{k}+\boldsymbol{u}_{k} \mathrm{E}_{k}^{m-g}\right) \\
\partial_{t}\left(\rho_{g} e_{g}\right)+\partial_{\boldsymbol{x}}\left(\rho_{g} e_{g} \boldsymbol{u}_{g}\right) & =-p \partial_{\boldsymbol{x}} \boldsymbol{u}_{g}-\partial_{\boldsymbol{x}} \mathbf{q}+\sum_{k}\left(-\mathrm{H}_{k}+\mathbf{F}_{k}\left(\boldsymbol{u}_{g}-\boldsymbol{u}_{k}\right)+h_{k} \mathrm{E}_{k}^{m-g}\right) \\
\partial_{t} n_{k}+\partial_{\boldsymbol{x}}\left(n_{k} \boldsymbol{u}_{k}\right) & =\mathrm{E}_{k+1}^{n}-\mathrm{E}_{k}^{n} \\
\partial_{t} m_{k}+\partial_{\boldsymbol{x}}\left(m_{k} \boldsymbol{u}_{k}\right) & =\mathrm{E}_{k+1}^{m}-\left(\mathrm{E}_{k}^{m}+\mathrm{E}_{k}^{m-g}\right) \\
\partial_{t}\left(m_{k} \boldsymbol{u}_{k}\right)+\partial_{\boldsymbol{x}}\left(m_{k} \boldsymbol{u}_{k} \otimes \boldsymbol{u}_{k}\right) & =\mathbf{F}_{k}+\boldsymbol{u}_{k+1} \mathrm{E}_{k+1}^{m}-\boldsymbol{u}_{k}\left(\mathrm{E}_{k}^{m}+\mathrm{E}_{k}^{m-g}\right) \\
\partial_{t}\left(m_{k} h_{k}\right)+\partial_{\boldsymbol{x}}\left(m_{k} h_{k} \boldsymbol{u}_{k}\right) & =\mathrm{H}_{k}+h_{k+1} \mathrm{E}_{k+1}^{m}-h_{k}\left(\mathrm{E}_{k}^{m}+\mathrm{E}_{k}^{m-g}\right)
\end{array}\right\} k \in \llbracket 1 ; N_{\mathrm{sec}} \rrbracket .
$$

This is referred to as the Multi-Fluid model in the following and is our baseline system of equations from now on.

\subsection{Time integration of the coupled system}

We apply operator splitting to Eq. (9), which allows one to use time integration schemes that are dedicated to the peculiarities of its different parts [32, 29]. The couplings can be rendered accurately if the operators and splitting sequence are defined correctly, and if the overall splitting time step $\Delta t$ is well chosen. A more complete discussion on splitting, as applied to coupled spray systems, can be found in [39]. In the present, we define the three operators illustrated in Fig. 2. These are the gas transport $\mathscr{T}_{g}$, section transport $\mathscr{T}_{k}$, where $k \in \llbracket 1 ; N_{\mathrm{sec}} \rrbracket$, and the coupling operator $\mathscr{C}$ that describes how fast the liquid and gas velocities converge locally to an equilibrium value in a way that conserves the system's momentum.

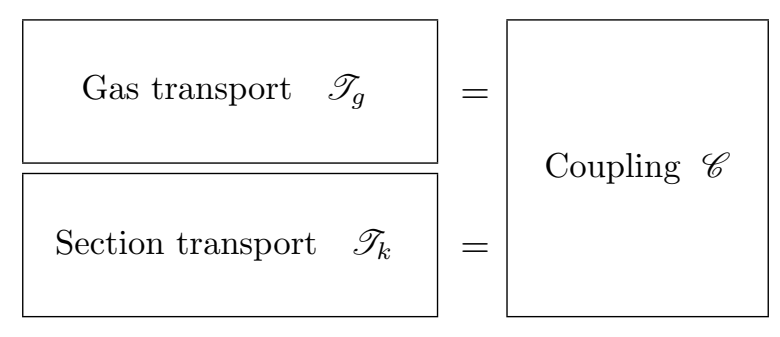

Figure 2: Schematic of the present splitting strategy.

The transport part of the gas phase, $\mathscr{T}_{g}$, reduces to the Navier-Stokes system. Either explicit, implicit, or mixed time integration strategies can be considered, with the usual constraints such as 
Courant-Friederichs-Lewy number (CFL) and Von-Neuman number (VNN) for this mixed hyperbolicparabolic set of equations. The coupling operator, $\mathscr{C}$, is a set of ODEs

$$
\left\{\begin{aligned}
\mathrm{d}_{t}\left(\rho_{g} Y_{l}\right) & =0, \\
\mathrm{~d}_{t}\left(\rho_{g} Y_{f}\right) & =\sum_{k} \mathrm{E}_{k}^{m-g} \\
\mathrm{~d}_{t}\left(\rho_{g} \boldsymbol{u}_{g}\right) & =\sum_{k}\left(-\mathbf{F}_{k}+\boldsymbol{u}_{k} \mathrm{E}_{k}^{m-g}\right) \\
\mathrm{d}_{t}\left(\rho_{g} e_{g}\right) & =\sum_{k}\left(-\mathrm{H}_{k}+\mathbf{F}_{k}\left(\boldsymbol{u}_{g}-\boldsymbol{u}_{k}\right)+h_{k} \mathrm{E}_{k}^{m-g}\right) \\
\mathrm{d}_{t} n_{k} & =\mathrm{E}_{k+1}^{n}-\mathrm{E}_{k}^{n} \\
\mathrm{~d}_{t} m_{k} & =\mathrm{E}_{k+1}^{m}-\left(\mathrm{E}_{k}^{m}+\mathrm{E}_{k}^{m-g}\right) \\
\mathrm{d}_{t}\left(m_{k} \boldsymbol{u}_{k}\right) & =\mathbf{F}_{k}+\boldsymbol{u}_{k+1} \mathrm{E}_{k+1}^{m}-\boldsymbol{u}_{k}\left(\mathrm{E}_{k}^{m}+\mathrm{E}_{k}^{m-g}\right) \\
\mathrm{d}_{t}\left(m_{k} h_{k}\right) & =\mathrm{H}_{k}+h_{k+1} \mathrm{E}_{k+1}^{m}-h_{k}\left(\mathrm{E}_{k}^{m}+\mathrm{E}_{k}^{m-g}\right)
\end{aligned}\right\} k \in \llbracket 1 ; N_{\mathrm{sec}} \rrbracket
$$

that can be integrated explicitly provided that its time scales are larger than the overall splitting time step $\Delta t$. Note that the characteristic time for the two-way coupled system with respect to drag gets shorter with a higher liquid loading. This time is locally of the order of

$$
\tau_{c}=\min _{k}\left(\frac{\tau^{\boldsymbol{u}}\left(r_{k}\right)}{1+m_{k} / \rho_{g}}\right)
$$

where $\tau^{\boldsymbol{u}}\left(r_{k}\right)$ is the characteristic time of the drag force $\mathbf{F}_{k}$. The same argument applies to heat transfer with $\tau^{T}\left(r_{k}\right)$ the characteristic time of the heat transfer $\mathrm{H}_{k}$, which leads to an additional constraint on $\tau_{c}$. As a consequence, the discrepancies introduced by spherical closures on momentum coupling do not have a significant impact in the regions where loading is high enough for the liquid to completely drive the dynamics. In these regions, the overall dynamics is dominated by the liquid so it is sufficient to maintain the velocities and temperatures close to equilibrium. To respect the coupling between the dense phase and gas phase, the splitting time step is chosen to be equal to the gas phase time step following a standard CFL constraint. Finally, the transported part of the disperse phase, $\mathscr{T}_{k}$, reduces to $N_{\text {sec }}$ systems

$$
\left\{\begin{array}{l}
\partial_{t} n_{k}+\partial_{\boldsymbol{x}}\left(n_{k} \boldsymbol{u}_{k}\right)=0 \\
\partial_{t} m_{k}+\partial_{\boldsymbol{x}}\left(m_{k} \boldsymbol{u}_{k}\right)=0 \\
\partial_{t}\left(m_{k} \boldsymbol{u}_{k}\right)+\partial_{\boldsymbol{x}}\left(m_{k} \boldsymbol{u}_{k} \otimes \boldsymbol{u}_{k}\right)=0 \\
\partial_{t}\left(m_{k} h_{k}\right)+\partial_{\boldsymbol{x}}\left(m_{k} h_{k} \boldsymbol{u}_{k}\right)=0
\end{array}\right.
$$

with a behavior equivalent to Eq. (3), though with an additional passive scalar.

\subsection{FSL for spray in splitting context}

The $N_{\text {sec }}$ systems (12) can be transported with the FSL method described in Section 2. In the context of operator splitting, the transport of each section is decoupled from the others so the adaptation of FSL to the Multi-Fluid model is straightforward, involving a different set of particles $p$ for each section $k$. High-order Multi-Fluid methods also require to deal with additional transported variables, here additional size moments (mass $m_{k}$ ) and temperature $T_{k}$, which is treated as a passive scalar $\phi_{k}$. The transport of the polydisperse spray is performed first with the scattering mapping

$$
\left(\begin{array}{c}
n_{k} \\
m_{k} \\
m_{k} \boldsymbol{u}_{k} \\
m_{k} \phi_{k}
\end{array}\right)\left(t, \boldsymbol{x}_{i}\right) \text { with }(i, k) \in \llbracket 1 ; N_{\text {cell }} \rrbracket \times \llbracket 1 ; N_{\text {sec }} \rrbracket \rightarrow\left(\begin{array}{c}
\boldsymbol{x}_{p} \\
n_{p} \\
m_{p} \\
\boldsymbol{u}_{p} \\
\phi_{p}
\end{array}\right)(t) \text { with } p \in \llbracket 1 ; N_{p} \rrbracket,
$$


where $\boldsymbol{x}_{p}=\boldsymbol{x}_{i}, n_{p}=n_{k}\left(t, \boldsymbol{x}_{i}\right), m_{p}=m_{k}\left(t, \boldsymbol{x}_{i}\right), \boldsymbol{u}_{p}=\boldsymbol{u}_{k}\left(t, \boldsymbol{x}_{i}\right), \phi_{p}=\phi_{k}\left(t, \boldsymbol{x}_{i}\right)$, and second with the gathering mapping

$$
\forall(i, k) \in \llbracket 1 ; N_{\text {cell }} \rrbracket \times \llbracket 1 ; N_{\text {sec }} \rrbracket, \quad\left(\begin{array}{c}
\boldsymbol{x}_{i} \\
n_{i, k}=\frac{1}{m_{i, k}} \sum_{p \in \Omega_{\boldsymbol{x}_{i}}} w_{p i} n_{p} \\
m_{i, k}=\sum_{p \in \Omega_{\boldsymbol{x}_{i}}} w_{p i} m_{p} \\
\boldsymbol{u}_{i, k}=\frac{1}{m_{i, k}} \sum_{p \in \Omega_{\boldsymbol{x}_{i}}} w_{p i} m_{p} \boldsymbol{u}_{p} \\
\phi_{i, k}=\frac{1}{m_{i, k}} \sum_{p \in \Omega_{\boldsymbol{x}_{i}}} w_{p i} m_{p} \phi_{p}
\end{array}\right) \rightarrow\left(\begin{array}{c}
n_{k} \\
m_{k} \\
m_{k} \boldsymbol{u}_{k} \\
m_{k} \phi_{k}
\end{array}\right)\left(t+\Delta t, \boldsymbol{x}_{i}\right),
$$

which involves parcels with $\boldsymbol{x}_{p}(t+\Delta t)$ in the $\Omega_{\boldsymbol{x}_{i}}$ ensembles to be associated to the relevant $\boldsymbol{x}_{i}$. This system is projected with the linear algorithm defined in Section 2.3. Since all the passive scalars are projected using convex combinations with linear, geometrically defined, positive coefficients, realizability is guaranteed whatever the moments. Finally, the cost in the Multi-Fluid context is known: the assumption of size-conditioned velocities allows to use only one parcel per location and per section so we have $N_{p}=N_{\text {cell }} \times N_{\text {sec }}$.

Using the algorithm described in Section 2.2, the full sequence including transport and splitting now reads as follows.

1a) Scatter is done according to the mapping of Eq. (13).

1b) Transport: $\boldsymbol{x}_{p}(t+\Delta t)=\boldsymbol{x}_{p}(t)+\boldsymbol{u}_{p}(t) \Delta t$. These $N_{p}$ trivial linear extrapolations exactly solve the transport part of the kinetic equation in the context of operator splitting.

2a) Gather (projection) according to the mapping of Eq. (14).

2b) Coupling (phase space evolution): Due to the above operator splitting, the remaining operators of Eq. (9) have to be solved. These operators sum up to coupling, which is detailed in Eq. (10). The Eulerian fields encounter a phase space evolution (due to drag, heating, evaporation, breakup, collisions) on $\Delta t$. At the end of this step, the Eulerian field is the approximated solution of Eq. (9) at time $t_{0}+\Delta t$. This splitting is a first order Lie splitting [32].

Step 1a) can be renewed in a cycle shown in Fig. 3, defining the Lagrangian state at $t+\Delta t$. So the splitting sequence can be summarized using symbolic operator notations:

$$
\boldsymbol{U}(t+\Delta t)=\mathscr{C} \prod_{k=1}^{N_{\text {sec }}}\left(\mathscr{T}_{k}\right) \mathscr{T}_{g} \boldsymbol{U}(t) \quad \text { with } \quad \boldsymbol{U}(t)=\left(\begin{array}{c}
\rho_{g} \\
\rho_{g} \boldsymbol{u}_{g} \\
\rho_{g} e_{g} \\
n_{k} \\
m_{k} \\
m_{k} \boldsymbol{u}_{k} \\
m_{k} T_{k}
\end{array}\right)(t)
$$

The key is to perform frequent projections of the mass and momentum on an Eulerian mesh in order to use the Eulerian fields for the coupling step (Euler-Euler coupling), and redefine the way the field is sampled by Lagrangian parcels. The projection/emission of Lagrangian parcels can be seen as a remeshing procedure, as used in vortex methods [70, 23].

\section{Implementation in an LES solver}

The FSL-LP scheme achieves realizable transport of the moments, whatever the size and velocity reconstructions are, so that no clipping is needed. This guarantees both accuracy and robustness as well as ease in handling the code and setting the configurations. This model and methodology have been implemented in an LES solver called RAPTOR and developed by Oefelein [74]. 


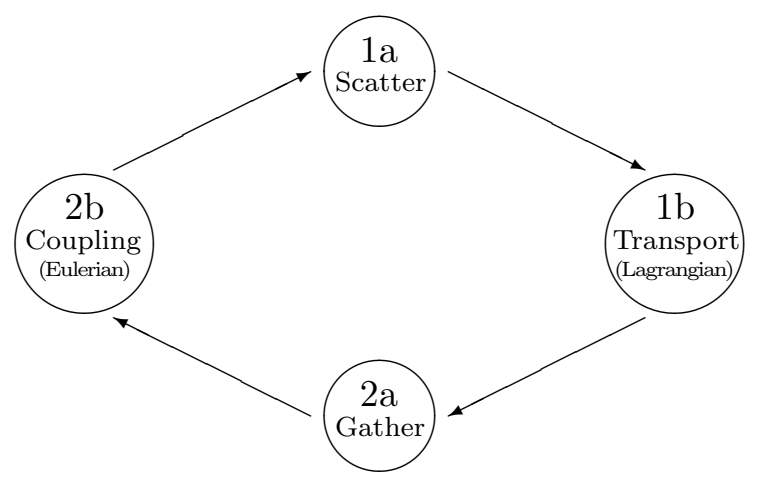

Figure 3: Schematic of the gather-scatter cycle as the algorithm for the FSL transport scheme.

\subsection{Splitting time step in high-fidelity LES strategy}

In the context of operator splitting, the transport part of the gas phase reduces to the NavierStokes system. The fluxes are computed on a staggered structured grid using a $3^{\text {rd }}$ order interpolation, limited with a blending function. Transport is time-integrated with an explicit method (RK4) [74]. An explicit integration with small time steps and a low dissipation flux scheme allows one to account accurately for both the convective and acoustics structures in the flow and requires, for convectiondominated flows $(\operatorname{Re} \gg 1)$, to observe the CFL constraint based on the fastest traveling wave, that is an acoustic wave

$$
\Delta t<\tau_{g}=\min \left(\frac{\Delta x}{\left|\boldsymbol{u}_{g}\right|+c}\right) .
$$

To preserve the coupling between the liquid and the gas phases, the splitting time step is chosen to be equal to the gas phase time step [39]. The coupling can be fast compared to the injection timescale, especially for small drops and for high loadings. But the fine spatial resolution of high-fidelity LES usually brings $\Delta t$ below $10^{-8}$ s so the gas timescales remain the stiffest and the gas CFL is the most restrictive stability constraint. Enforcing a splitting time step smaller than the gas timescale is a criterion of accuracy of the splitting [39]. The mass, momentum, and heat transfers can then be integrated explicitly.

\subsection{Phase space integrator verification}

To time-integrate the coupling operator $\mathscr{C}$, we use an explicit, Runge-Kutta 4 (RK4) scheme. We perform a quantitative verification of the two-way coupling solver based on the characteristic time and equilibrium velocity of a 0D monodisperse spray. Results are shown for various droplet sizes $r_{k}$ in Fig. 4 (left) and for various droplet loadings in Fig. 4 (right). The reference times and equilibrium velocities are computed from the following analytical formulae

$$
\tau_{c}^{\boldsymbol{u}}=\frac{\tau^{\boldsymbol{u}}\left(r_{k}\right)}{1+m_{k} / \rho_{g}} \quad \text { and } \quad \boldsymbol{u}_{\mathrm{eq}}=\frac{\rho_{g} \boldsymbol{u}_{g}+m_{k} \boldsymbol{u}_{k}}{\rho_{g}+m_{k}}
$$

and the numerical results compare well. The verification of the heat exchange terms is not presented here but the terms are coded similarly. The verification of the evaporation terms is not presented. The integration module computes all three sources simultaneously, in the same RK4 sequence, therefore achieving a full coupling of the phase space.

\section{Verification of the FSL-LP transport scheme}

We now perform verifications for the FSL-LP transport scheme using 1D cartesian cases, a 2D structured case, and a 0D case with sources. This allows us to quantify the order of the method, to assess its qualities for practical cases, and to verify its constitutive modules. 

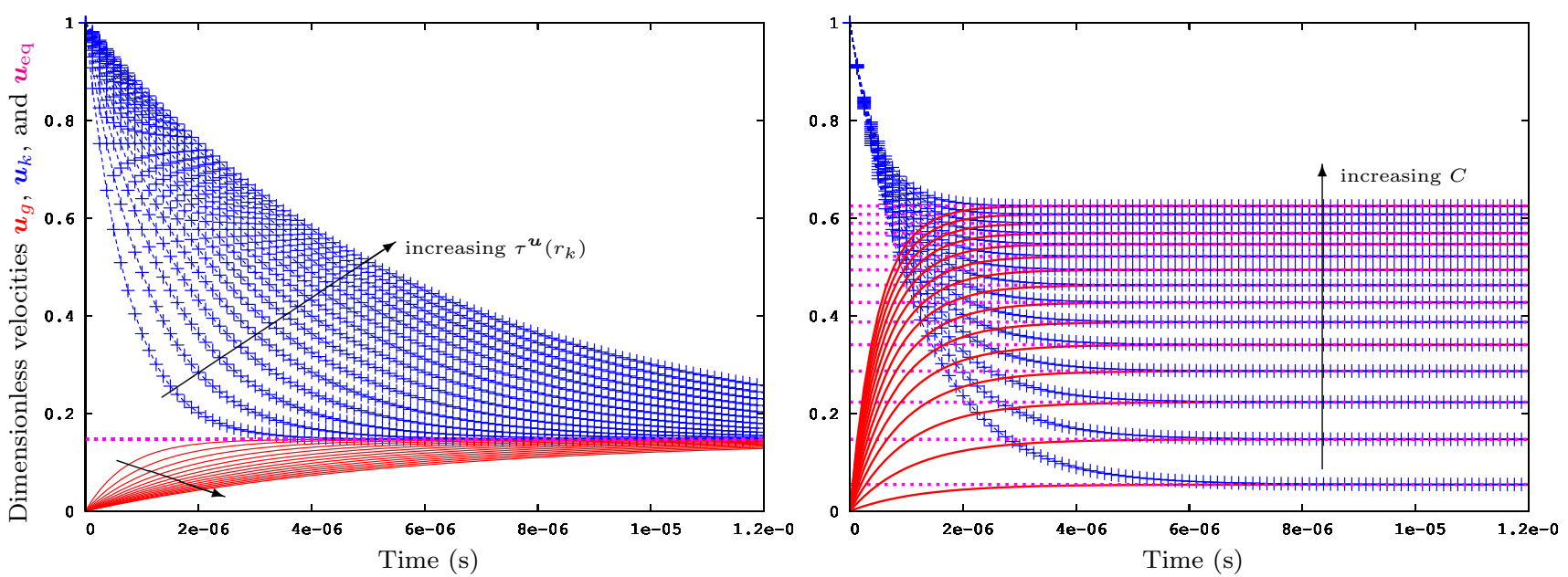

Figure 4: Computations of drag with two-way coupling (0D) giving quantitative verification of the phase space integrator - Left: Various droplet sizes/characteristic times with $C=0.17$; Right: Various mass loadings with $\tau_{c}^{u}=10^{-6} \mathrm{~s}-$ Solid: $\boldsymbol{u}_{g} ;-+-: \boldsymbol{u}_{k} ; \cdots \cdots: \boldsymbol{u}_{\text {eq }}$.

\section{1. $1 D$ linear advection}

We consider the case of the advection of a smooth profile at uniform velocity. This study compliments the analytical assessment of FSL-LP performed in Section Appendix A. A Gaussian density profile is selected for its smoothness and the reference solution can be devised analytically, thanks to the linearity of advection. The goal is to assess the actual order of the FSL-LP transport scheme. A regular and a stretched mesh are considered to discretize the segment $\left[0, L_{x}\right]$ with $L_{x}=1$. The stretched mesh is stretched towards $x>0$ according to $x_{i}=L_{x}\left(i / N_{x}\right)^{2}$. The convergence of general semi-Lagrangian methods is a topic under study $[56,55]$, but, in the present case of FSL with a linear projection, this matter is dominated by the accuracy of the projection on the stretched grid. We here propose an empirical verification on a linear advection case. The error is assessed using the $L^{1}$ norm

$$
E_{N_{x}}=\frac{1}{N_{x}} \sum_{i=1}^{N_{x}}\left|m_{N_{x}}\left(x_{i}\right)-m_{\mathrm{ref}}\left(x_{i}\right)\right| .
$$

Results are given in Fig. 5 after one time step with a fixed $u \Delta t=1 / 20$ for all levels of refinement. This proves that the method is convergent regardless of the type of mesh. The slopes indicate a local error of order 2 for both types of mesh. This means that, for a fixed CFL, the global order of the method would be 1 because of the errors introduced by the number of time steps linearly increasing with $N_{x}$. Note however that the method can be used at arbitrary CFL. Note also that there exist, in the regular mesh case, some discretizations for which the error dramatically drops (here by 6 orders of magnitude). This corresponds to integer CFL numbers, for which the projection is exact. As a conclusion, the method is convergent with the expected order on both Cartesian and stretched meshes.

\subsection{D PGD convection with vacuum and shock formation}

We now consider a case with convection; i.e., a type of transport where the velocity information is transported together with the quantity of interest (here mass) as opposed to advection where velocity is exogenous. We actually examine the ability of FSL-LP to deal with the full physics of PGD. To do so, we take the first test problem defined by Bouchut et al. [16]. It is a 1D problem featuring a velocity discontinuity and a velocity gradient which generates density discontinuities and vacuum. The initial conditions are

$$
\rho^{0}(x)=0.5, \quad u^{0}(x)=\left\{\begin{array}{cc}
-0.5, & x<-0.5, \\
0.4, & -0.5<x<0, \\
0.4-x, & 0<x<0.8, \\
-0.4, & x>0.8 .
\end{array}\right.
$$




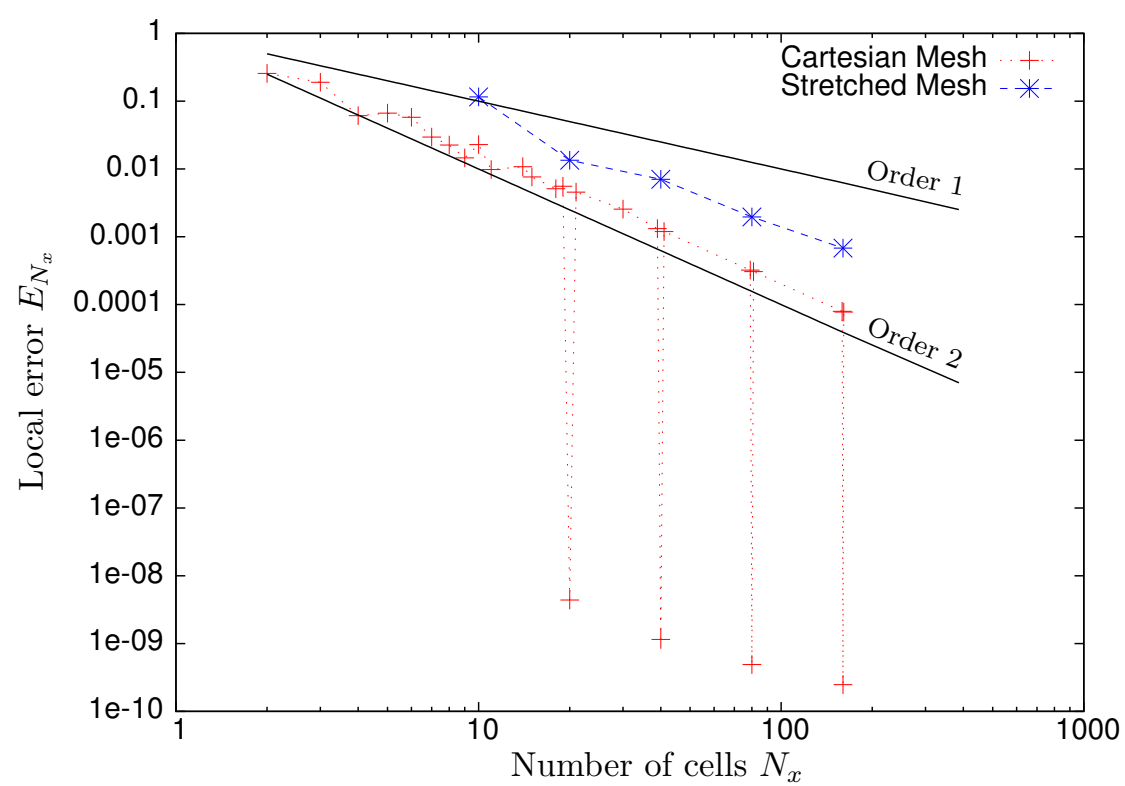

Figure 5: Convergence of the gather-scatter method on 1D advection of a Gaussian profile.

We compare in Fig. 6 the FSL-LP results to the analytical solution provided by the authors. The velocity field is accurately rendered and the density field appears slightly less smoothed out at discontinuities compared to Bouchut et al's results (not reproduced) obtained with their first order Godunov and Kinetic schemes. We observe the same spike at the zero-velocity (sonic) point, analyzed in [15]. The $\rho=1$ plateau is underestimated for mass conservation reasons, the missing mass being accumulated in the spike, as observed in the reference computation. As a conclusion, the FSL-LP transport scheme is fit to describe the peculiarities of PGD.
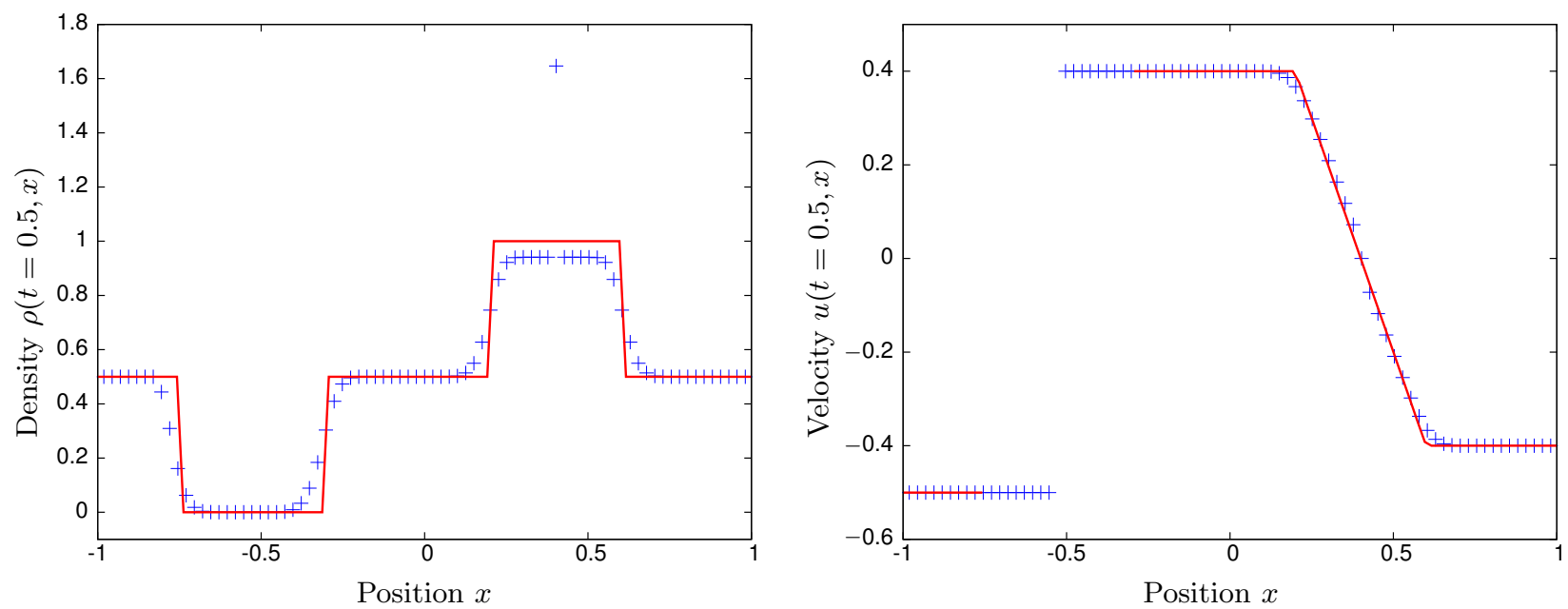

Figure 6: Solution at $t=0.5$ of Bouchut et al.'s "numerical test I". Exact solution (line) and FSL-LP scheme with $\Delta x=0.025$ and $\Delta t / \Delta x=5 / 3(+)$.

\subsection{Convection on a stretched structured mesh}

We now consider another problem of convection in the limit where negligible trajectory crossings happen. Convection is handled non-linearly by the FSL method due to momentum averaging at the cell centers. But in the absence of trajectory crossings the assumed equivalence between the kinetic description and the PGD model should hold. In other words the new method should compare well with reference DSMC if and only if negligible trajectory crossing occurs. 
A disperse phase jet is computed starting from the so-called Downstream Inflow Turbulent Boundary Conditions (DITurBC). It is a prescribed boundary condition used to emulate the outcome of primary atomization of a fuel spray for piston engine simulations [71]. The resulting cloud is transient as it penetrates the resolved domain, non-uniform, polydisperse, and has a size-conditioned velocity distribution. In our test case, the droplet cloud is one-way coupled to a prescribed gas field through a local drag source term. The same prescribed inlet condition is used to compute a reference DSMC solution. The choice of one-way coupling is motivated for verification. The same gas field can then be taken for both formalisms and it is a classical first step for comparisons between Eulerian-Eulerian and Eulerian-Lagrangian approaches [29, 49]. The gas field is a slice of a round jet that has been computed offline and is discretized over a self-similarly stretched grid that is typical for these types of jets. For the computation of the coupling; i.e., drag evaluation, the gas velocity values are interpolated at particle locations, which are arbitrary for DSMC and regularly spaced within the stretched grid cells for FSL. The same time step is used, being close to the particle CFL constraint (convective) based on the gas grid at the inlet. The number of degrees of freedom to describe the disperse phase is taken to be the same for both methods with DSMC using 2 million parcels (sampling random initial locations and droplet sizes) and with FSL-LP using 10 sections (to discretize size phase space) and being ultimately projected on 0.2 million cell centers.

Results are given in Fig. 7. The two simulations compare very well, which proves that there is little trajectory crossing so that the monokinetic assumption in FSL-LP is satisfactory. The cost of FSL-LP is affordable. It computes faster than the DSMC with the same number of parcels, but none of the two codes have been profiled to check if they correspond to competitive implementations. Still this advocates for a good performance of FSL-LP. The DSMC exhibits noise on the spatial fields in the depleted regions, while FSL is statistically converged by construction. In return, the FSL-LP fields are smoother due to numerical diffusion. In terms of the description of polydispersity, the same conclusions are expected, with DSMC featuring noise on the size and velocity distributions while MultiFluid FSL-LP provides a statistically converged description of the phase space by construction. We do not present results on the size phase space for the sake of a fair comparison between size sampling and size binning. The present test case has no evaporation, so that the Multi-Fluid approach is favored and will not introduce numerical diffusion in the size phase space.

To gain a clearer view of the drawbacks of stochastic methods on particle transport, it can be seen in Fig. 8 that the number of parcels for DSMC is very non-uniform. These non-uniformities are driven by the Monte-Carlo initial conditions and they are very difficult to predict and to correct. The present non-uniformities result in waste of computational resource in the over-sampled regions and in noise in the under-sampled regions. Moreover, Fig. 8 illustrates the load-balancing issues that will arise in a parallel execution of DSMC. These issues of Monte-Carlo convergence and load-balancing do not arise with the FSL scheme, where the degrees of freedom are linked to the number of cells, which is predictable and controllable. This case demonstrates that FSL-LP is verified. Deterministic solvers are recommended to handle a polydisperse spray in a complex gas field, whenever low (null) noise content is a strong requirement.

\subsection{Conclusions on FSL-LP verification}

The FSL-LP transport scheme has been checked with respect to accuracy on linear and non-linear PGD transport. The one-way coupled 2D case has exhibited the cost/accuracy trade-off as compared to DSMC. FSL-LP has proved consistency, accuracy, robustness, and load-balancing relevance to parallel computations.

- It has no noise and a naturally balanced and predictible load as opposed to DSMC.

- Its implementation is simple and easily extended to spray methods with more moments with guaranteed realizability.

- Its low space order is balanced by excellent conservation and robustness properties.

To be exploited for large and complex spray applications, the method must now be checked in two-way coupled and dense regimes. 


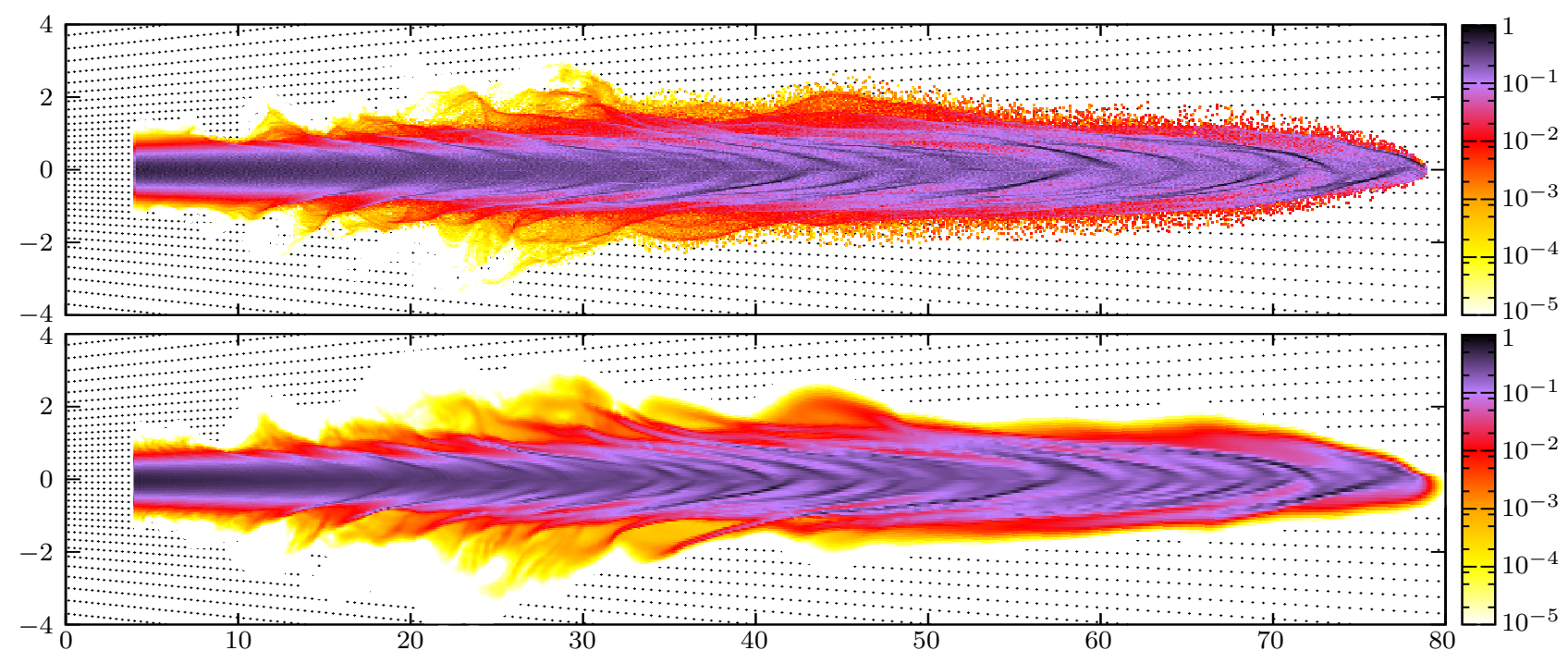

Figure 7: Direct fuel injection spray prescribed using DITurBC in 2D and with one-way coupling - Fuel mass concentration normalized by $\rho_{l}$ with Lagrangian DSMC (top, 2Mparcels) and Hybrid Eulerian-Lagrangian (bottom, 200kcells $\times 10$ sections). The black dots are the locations of the gas field cell centers.

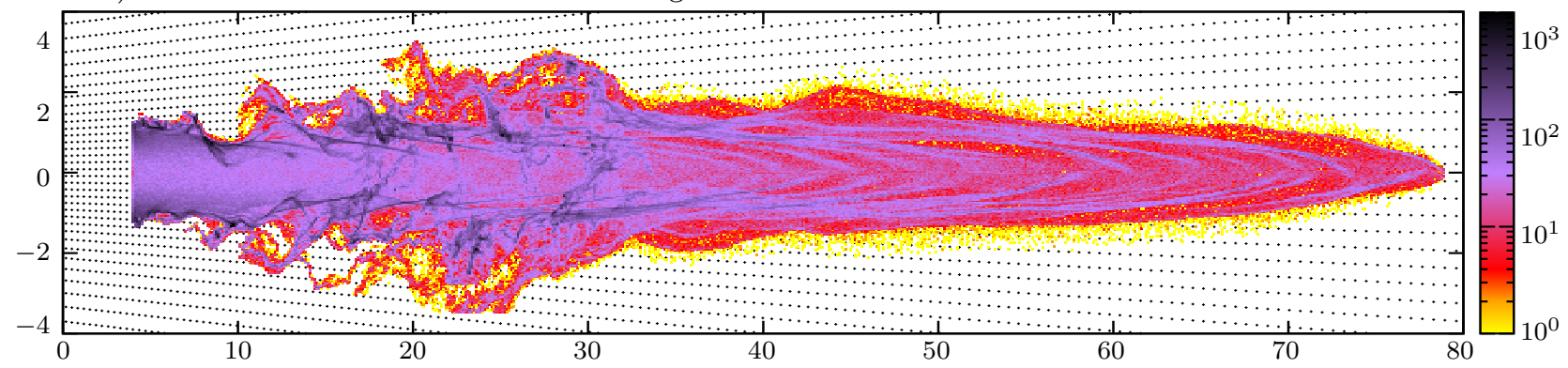

Figure 8: Number of numerical parcels per cell with Lagrangian DSMC for the 2D one-way coupled case.

\section{Extension of spray model to dense regime}

The extension of the disperse phase model, based on kinetic theory with assumption of spherical particles, to the near-nozzle region has not been validated yet. To describe a dense liquid core, it is reasonable to use a moment method that conserves mass and momentum of liquid but the use of spherical closures, the incorrect compressibility, and the lack of resolved surface tension are questionnable. In the context of direct fuel injection however, the spray is injected with a high amount of momentum and a high Weber number so that accounting for liquid inertia may be sufficient.

\subsection{Argument on dense coupling timescales}

A local characteristic drag time, as introduced in Section 4.2, stems from eigenvalue analysis of the two-way coupled momentum equations

$$
\tau_{c}^{\boldsymbol{u}}(t, \boldsymbol{x})=\frac{\tau^{\boldsymbol{u}}\left(r_{k}(t, \boldsymbol{x})\right)}{1+m_{k}(t, \boldsymbol{x}) / \rho_{g}(t, \boldsymbol{x})}
$$

where $\tau^{\boldsymbol{u}}(r)$ is the characteristic time of the drag force $\mathbf{F}$ and the local drop radius $r_{k}$ can be computed from $n_{k}$ and $m_{k}$. The same argument applies to a coupled heat transfer time $\tau_{c}^{T}$ by using $\tau^{T}(r)$ the characteristic time of $\mathrm{H}$. So the characteristic times for the two-way coupled system get shorter with higher liquid loading. This implies that the discrepancies introduced by spherical closures do not have a significant impact in the regions where loading is high. In these regions, the overall dynamics should be dominated by the liquid. And the coupling does actually maintain the velocities and temperatures close to equilibrium, provided that the corresponding characteristic timescales will be small. 


\subsection{Validation on near-nozzle flow}

We compare our approach to a reference solver, solving the Navier-Stokes equation governing both the liquid and the gas and accounting for sharp interfaces and surface tension. A set of calculations have been performed on a case of direct fuel injection (spray A conditions, extensively described in Section 7) with both the Multi-Fluid model with the FSL-LP scheme and the Coupled Level-Set Volume of Fluid (CLSVOF) approach [59,3]. The CLSVOF approach is a state-of-the-art interfacecapturing method which accounts for the sharp interface allowing the conservation of volume and the accurate computation of the surface tension. It is classically used for the direct numerical simulation (DNS) of two-phase flows and it provides a reference on momentum coupling between liquid and gas.

The comparison is conducted at an early stage of injection, namely when the liquid trajectory is still weakly influenced by the surrounding gas. We choose to look at an instant $3.7 \mu$ s after the start of injection (ASOI) with a simple plug flow as a model boundary condition for the liquid; i.e., a laminar profile with no boundary layer. Results are given in Fig. 9. The use of a tailored operator splitting has allowed robust and accurate handling of two-way coupling, including when mass loading is high. Strong entrainment is created in the chamber because the liquid mass fraction and momentum ratio are high. The two approaches agree very well on the gas flow field and on the inertial behavior of the liquid core. The penetration lengths match to within $1 \%$. The only difference is that the tip of the jet simulated with Multi-Fluid has a more elongated shape presumably due to the lack of surface tension in the current model.

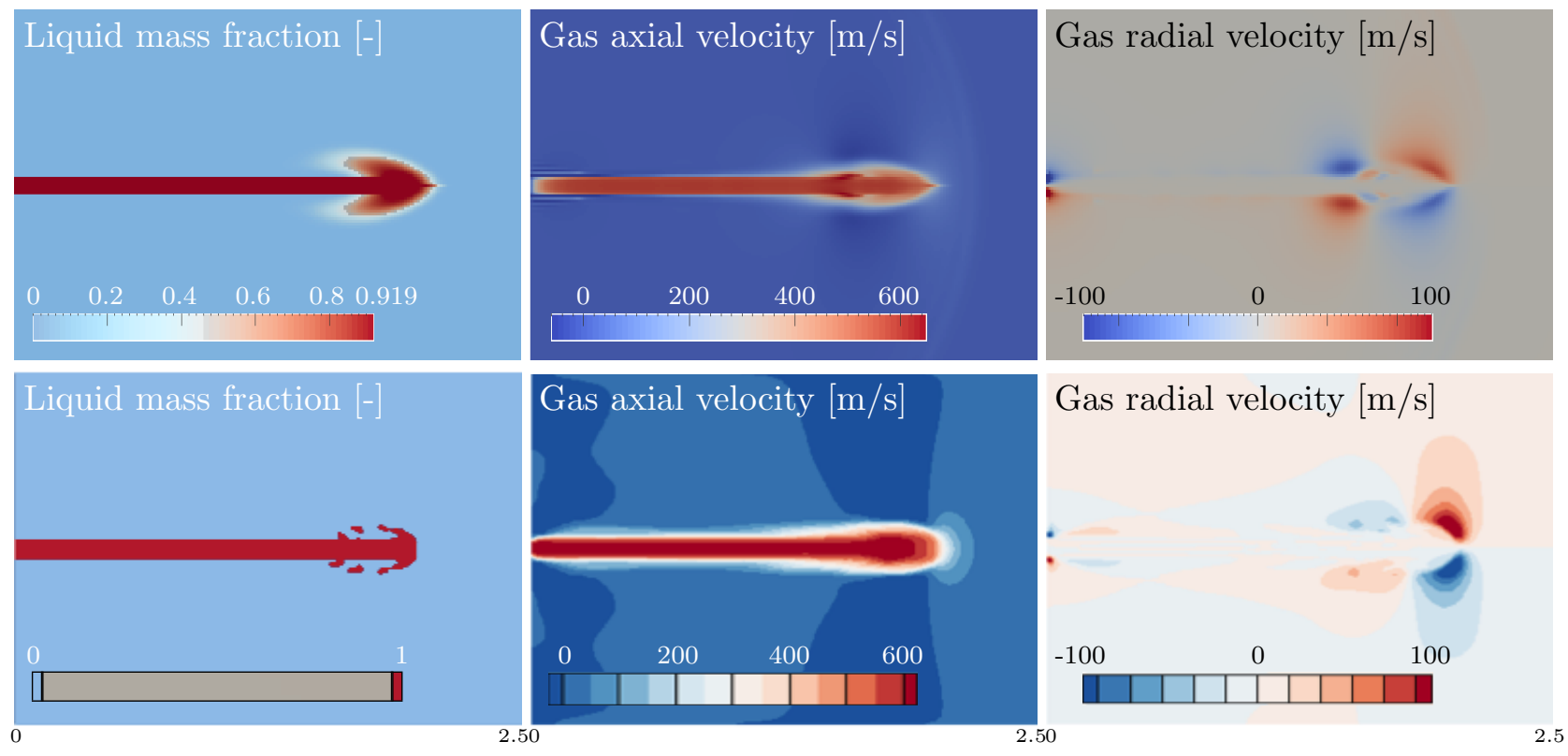

Figure 9: Multi-Fluid (top) and CLSVOF (bottom) injection with a laminar boundary condition at $3.7 \mu$ s ASOI (center plane slices) - Lengths are in $\mathrm{mm}$.

\subsection{Prospects for LES of atomization}

We have demonstrated that our two-phase solver behaves well in the near-nozzle region where the liquid volume fraction is close to 1 . There, the sensitivity of the transfers to the prescribed droplet size is low. However, the approach has five potential limitations for the description of direct fuel injection. A) The drop sizes, which will play a key role in the dilute region, stem from small scale interactions (atomization) that are not captured. Therefore a sound drop size distribution has to be prescribed. B) The drop ejection velocities at the edge of the core could play a role, as well as the size and velocity fluctuations in space and time so that an atomization sub-model could be needed in the bulk of the computation. C) The collision, coalescence, and break-up models will also play a role in some regions with intermediate volume fractions, transitioning from dense to dilute spray. D) The 
compressibility of the liquid in the core is not properly rendered by our disperse phase description and could play a role; e.g., in the formation of the mushroom shaped-tip observed numerically for plug-flow initial profiles. E) Surface tension is not accounted for in our approach. While it is known for driving large scale dynamics (Rayleigh-Taylor, Rayleigh-Plateau, jet and sheet flapping) at low Reynolds and Weber numbers, it has not been checked whether surface tension effects can be neglected at large scale in direct fuel injection or not.

\section{Applied computations}

A realistic spray case for direct fuel injection is now considered. We show numerical results of high fidelity simulations to clarify the potential of the method to act as a research tool on reacting spray physics and engine development. The strategy has been proposed and justified for engine sprays in $[36]$.

\subsection{Near-nozzle monodisperse Spray A case}

We consider the conditions of the so-called Spray A experiment, as taken from the Engine Combustion Network (ECN). Spray A has been extensively studied and we refer the reader to Bardi et al. [5] and subsequent publications for more details. In its non-reacting version, a hot pressurized chamber is filled with pure nitrogen $\left(\mathrm{N}_{2}, T_{g}=900 \mathrm{~K}, P_{g}=60 \mathrm{bar}, \rho_{g}=22.4 \mathrm{~kg} / \mathrm{m}^{3}\right)$. The fuel, n-dodecane $\left(\mathrm{C}_{12} \mathrm{H}_{26}, \rho_{l}=702 \mathrm{~kg} / \mathrm{m}^{3}, T_{l}=363 \mathrm{~K}\right)$, is injected as a round jet of liquid drops at $\boldsymbol{u}_{k}\left(t, \boldsymbol{x}_{0}\right)=600 \mathrm{~m} / \mathrm{s}$, that is close to sonic speed, from the single injector $(d=90 \mu \mathrm{m})$. At present, the nozzle internal flow is not computed. Instead a laminar plug-flow profile is used, which creates a discrepancy with experimental spray angles but does not interfere with the present feasibility demonstration. The geometry is a rectangular box of dimensions $9.6 \times 3.2 \times 3.2 \mathrm{~mm}^{3}$, which is shorter than the typical length of the steady state spray as the study is focused on the near-nozzle region of the jet. A cartesian mesh of $768 \times 256 \times 256$ cells is used, which translates to 7 cells per nozzle diameter. For the drag force, heat transfer, and evaporation characteristic times, the equivalent drop radius is taken to be $r=2 \mu \mathrm{m}$, which leads to coupling times that are close to and above the computational time step $\Delta t$. The latter is actually constrained by the Navier-Stokes solver CFL criterion based on the characteristic time of the fastest wave $\tau_{g}$, which yields $\Delta t=8 \mathrm{~ns}$. The droplet size is prescribed small to test the overall quality of the numerical strategy in the presence of stiff transfer terms and strong coupling. It also corresponds to the possible droplet sizes produced by Spray A atomization as reported by preliminary X-ray and microscopy measurements reported by ECN. All the characteristics of this computation are summed up in the left column of Table 1. LES is employed due to the high Reynolds number of the case. The computation is performed using a single-phase LES dynamic model [74]. The transfers between the two phases are computed from the resolved quantities and no dedicated two-phase LES model is used, as discussed in Section 3.1. So the droplet transport is not directly influenced by the gas subgrid turbulence and the gas is not influenced by the droplets' subgrid motion. The authors agree that turbulent dispersion is important in sprays, but in this dense spray context no satisfactory model exists and the physics is not well known. The method has already been used for the study of momentum coupling in spray injection [35] and for preliminary studies on autoignition [36]. Here we analyze realistic spray results to prove the feasibility of the strategy for the simulation of dense sprays.

Results are shown in Fig. 10 for two cases where evaporation is turned off and turned on respectively. Since the initial mass loading ratio is $C=\rho_{l} / \rho_{g}=31$, we can conclude on the remarkable robustness of the code. Moreover, since the gas density goes as low as $\rho_{g}=4 \mathrm{~kg} / \mathrm{m}^{3}$ at the inlet boundary due to entrainment, the mass loading ratio $C$ can locally increase above 150 , which brings the characteristic time of the two-way coupled drag system close to $\tau_{c}^{\boldsymbol{u}}=8 \mathrm{~ns}$. These results demonstrate the efficiency of the spray strategy. The FSL-LP scheme overcomes the peculiarities of the disperse phase transport model and the tailored splitting accurately enforces the strong two-way coupling between the two phases. The low dissipation of the code, from the Navier-Stokes solver to the disperse phase solver and coupling scheme, allows one to resolve a significant amount of the turbulence induced by the jet. This matches the main scope of such computations, which is to capture the intermediate 


\begin{tabular}{l|cc} 
& Near-nozzle monodisperse & Polydisperse reacting \\
\hline Liquid density & $\rho_{l}=702 \mathrm{~kg} / \mathrm{m}^{3}$ & $702 \mathrm{~kg} / \mathrm{m}^{3}$ \\
Liquid temperature & $T_{l}=363 \mathrm{~K}$ & $363 \mathrm{~K}$ \\
\hline Chamber pressure & $p_{g}=60 / 80 \mathrm{bar}$ & $60 \mathrm{bar}$ \\
Chamber temperature & $T_{g}=900 / 1200 \mathrm{~K}$ & $900 \mathrm{~K}$ \\
Gas composition & $\mathrm{N}_{2}$ & Preheating residuals \\
Gas density & $\rho_{g}=22.4 \mathrm{~kg} / \mathrm{m}^{3}$ & $22.8 \mathrm{~kg} / \mathrm{m}^{3}$ \\
Gas viscosity & $\mu_{g}=4.32 \times 10^{-5} \mathrm{~Pa} . \mathrm{s}$ & $4.32 \times 10^{-5} \mathrm{Pa.s}$ \\
\hline Nozzle diameter & $d=90 \mu \mathrm{m}$ & $90 \mu \mathrm{m}$ \\
Inlet velocity & $\boldsymbol{u}_{k}\left(t, \boldsymbol{x}_{0}\right)=U_{l}=600 \mathrm{~m} / \mathrm{s}$ & $600 \mathrm{~m} / \mathrm{s}$ \\
Jet Reynolds number & $\operatorname{Re}_{g}=\frac{\rho_{g} U_{l} d}{\mu_{g}}=28000$ & 28000 \\
\hline Initial droplet radius & $r_{p}=2 \mu \mathrm{m}$ & $\mathrm{see} \mathrm{Table} 2$ \\
Drag characteristic time & $\tau_{p}^{u}=\frac{\rho_{l} r_{p}^{2}}{18 \mu_{g}}=14.4 \times 10^{-6} \mathrm{~s}$ & multiple times \\
\hline Domain size & $L_{x} \times L_{y} \times L_{z}=9.6 \times 3.2 \times 3.2 \mathrm{~mm}^{3}$ & $54 \times 10.8 \times 10.8 \mathrm{~mm}^{3}$ \\
Mesh & $N_{x} \times N_{y} \times N_{z}=768 \times 256 \times 256$ & $1200 \times 240 \times 240$ \\
Time step & $\Delta t=0.008 \mu \mathrm{s}$ & $0.040 \mu \mathrm{s}$ \\
Wall clock time@1600 CPUs & $\mathrm{WCT}<1$ day & 6 days
\end{tabular}

Table 1: Characteristics of the Spray A computations.

scale structures. These structures are indeed believed to participate in driving the mixing, ignition, and combustion processes.

We show in Fig. 11 the gas temperature and fuel vapor mass fraction after $t_{2}=60 \mu \mathrm{m}$. The conditions are hotter $\left(T_{g}=1200 \mathrm{~K}\right)$ and correspond to a case where ignition takes place earlier and closer to the injector compared to the $900 \mathrm{~K}$-case. The fuel vapor mass fraction, together with the level of turbulence and the temperature of the mixture, is considered to be a driver of autoignition [65, 54, 36]. The present results show the strong unevenness of the fuel vapor mass fraction and are promising for analyzing autoignition, its mechanisms, and its statistics, which is one of the long term goals of Diesel injection studies.

\subsection{Parallel assessment: strong scaling test}

The above case (Section 7.1) is considered for a strong scaling test. We recall it is a monodisperse two-phase Diesel spray on a structured mesh with 50-million cells. The test is executed on a cluster of AMD Opteron 6174 with 2688 available cores, grouped into nodes of $4 \times 12$ Magny-Cours cores. The computations are run until the jet fully reaches the output of the box in order to maximize the number of parcels. Such computations give a fair representation of the cost, since the latter is dependent on the number of cells where spray is present. Choosing an uncommon approach, we also included the $\mathrm{I} / \mathrm{O}$ in the quantification of the cost. This penalizes at most the parallel efficiency but allows to assess the method for its relevance to practical simulations.

All the gas and disperse phase fields are output every 20 time steps, which represents about $15 \%$ of the overall computational time. The output frequency is high compared to what is required for standard CFD analysis. This choice has two origins: i) to allow more flexibility during the computation setup and exploratory phase, and ii) to produce high framerate movies. The scaling results are given for the overall cost in Fig. 12 and they prove to be satisfactory. The strategy is qualified for massively parallel computations. Further analysis shows that the computational cost of the disperse phase operators (one section transport and coupling) is comparable though lower than that of the gas transport, as expected since multicomponent Navier-Stokes equations feature more operators.

We highlight that we have made no particular optimization for parallel computing, leveraging both the performance of the existing Navier-Stokes solver in RAPTOR [74] and the good load balance of the FSL-LP method as regards domain decomposition. As a conclusion, the overall parallel efficiency 

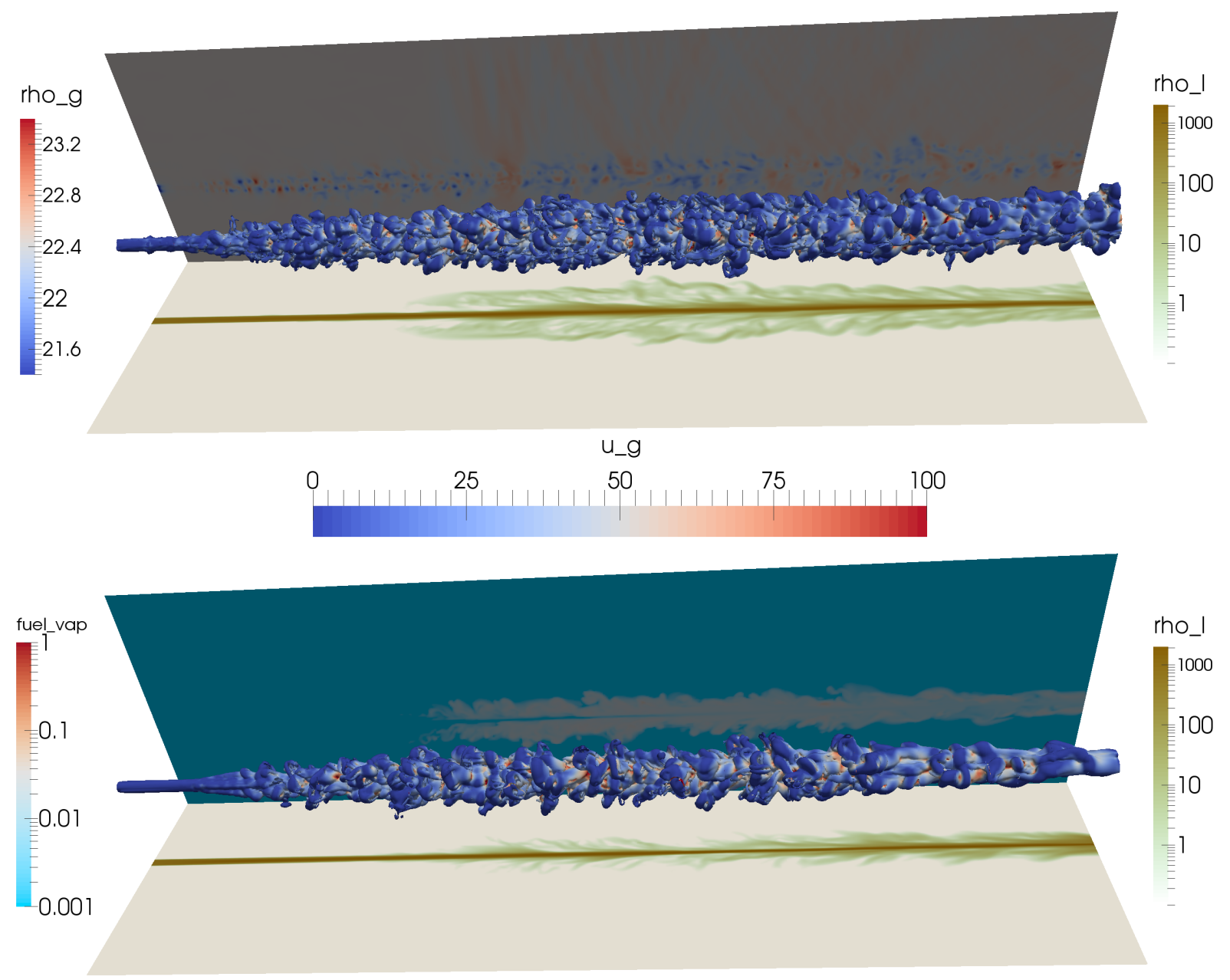

Figure 10: Near-nozzle monodisperse spray A case $\left(T_{g}=900 \mathrm{~K}\right)$ after $t_{1}=28 \mu \mathrm{s}$. Isocontour of gas vorticity norm colored by gas axial velocity $u_{g}$. Bottom plane shows the liquid density at horizontal centerplane slice. Top: Case without evaporation, back plane showing gas density at vertical centerplane slice; Bottom: Case with evaporation, back plane showing fuel vapor mass fraction at vertical centerplane slice.

of RAPTOR, when coupled with the Multi-Fluid solver, is excellent even with frequent outputs. As a result, with 1600 cores, which is a commonly affordable number of cores nowadays, the wall clock time for such a large test case is less than a day of computation, as stated in Table 1.

\subsection{Reacting polydisperse Spray A case}

To demonstrate the full modeling strategy, the reacting version of Spray A is finally presented with a polydisperse model for the disperse phase. According to Spray A specifications, the chamber $\left(T_{g}=900 \mathrm{~K}, P_{g}=6 \mathrm{MPa}\right)$ is filled with a mixture of the burnt gases left by the preheating with molar fractions $\mathrm{O}_{2}: 15.00 \%, \mathrm{~N}_{2}: 75.15 \%, \mathrm{CO}_{2}: 6.22 \%, \mathrm{H}_{2} \mathrm{O}: 3.62 \%$, and density $\rho_{g}=22.8 \mathrm{~kg} / \mathrm{m}^{3}$. The chemical reaction model is a two-step mechanism optimized for autoignition through statistical calibration [54]. The geometry is now a larger box of size $54 \times 10.8 \times 10.8 \mathrm{~mm}^{3}$ able to include the entire spray region at typical auto-ignition time. It is discretized as a cartesian mesh of $1200 \times 240 \times 240$ cells, which translates to 2 cells per nozzle diameter. The fuel is still n-dodecane $\left(\mathrm{C}_{12} \mathrm{H}_{26}, \rho_{l}=702 \mathrm{~kg} / \mathrm{m}^{3}\right.$, $\left.T_{l}=363 \mathrm{~K}\right)$ injected as a round jet of liquid drops gathered in several sections $k$ at a speed of $\boldsymbol{u}_{l}\left(t, \boldsymbol{x}_{0}\right)=600 \mathrm{~m} / \mathrm{s}$ from a single injector $\left(d_{\mathrm{inj}}=90 \mu \mathrm{m}\right)$. All the characteristics of this computation are summed up in the right column of Table 1.

For the fuel jet, a laminar plug-flow profile is used with a realistic velocity ramp to match the measured rate of injection [5]. An angle $\alpha_{0}=6 \mathrm{deg}$ between the injected drop velocities and the 

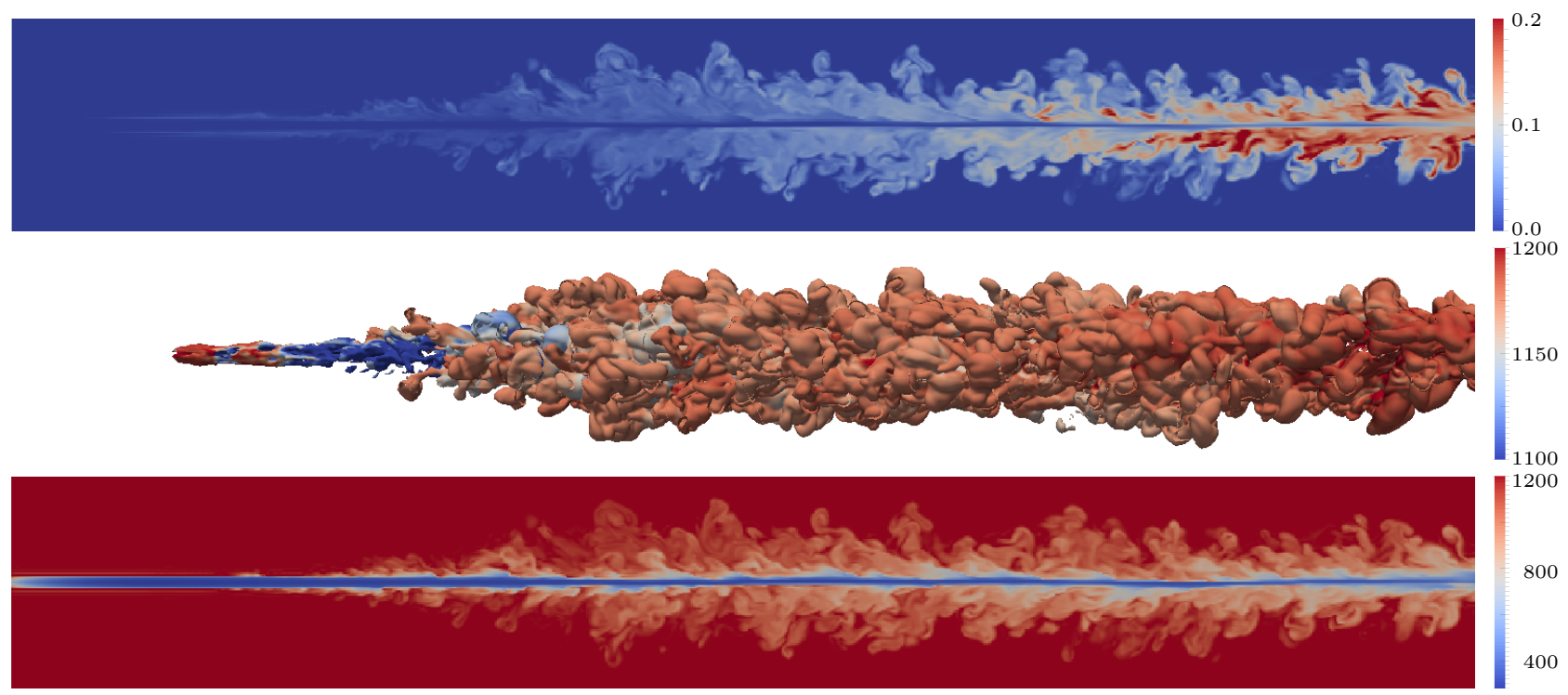

Figure 11: Near-nozzle monodisperse spray A case $\left(T_{g}=1200 \mathrm{~K}\right)$ with evaporation after $t_{2}=60 \mu \mathrm{s}$. Top: Fuel vapor mass fraction at centerplane; Middle: Fuel vapor isocontour with $Y_{\mathrm{C}_{12} \mathrm{H}_{26}}=0.01$; Bottom: Gas temperature at centerplane.

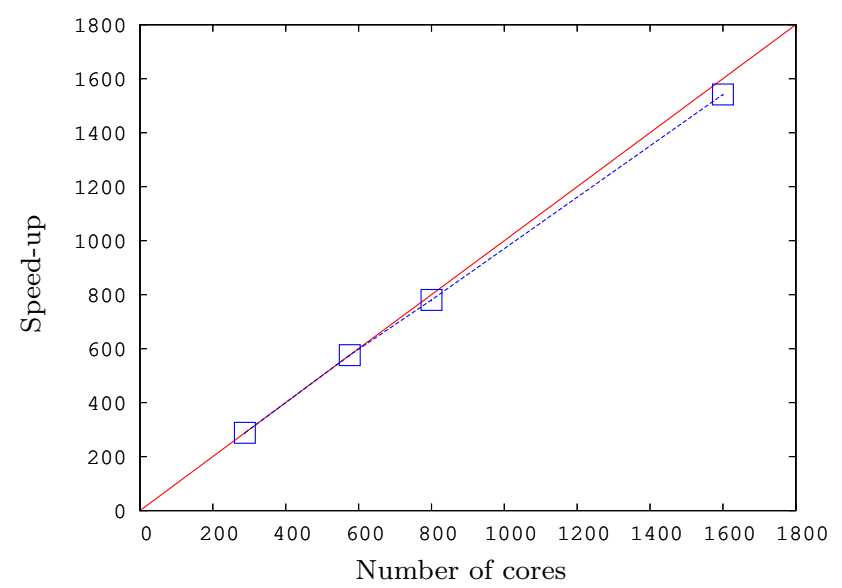

Figure 12: Strong scaling test on a cluster of AMD Opteron $6174(4 \times 12$ cores per node). $\square$ : present; line: ideal.

injection axis is prescribed so that drop trajectories spread conically from the axis. This one parameter comes from a simplified approach to describe the spreading of the spray due to turbulence and early atomization in order to match the spray angle. Particular care has been taken in prescribing the drop sizes, according to the first step of the discussion in Section 6.3. An average size distribution has been extracted from a DNS of atomization performed at the same engine conditions, without evaporation, and with a compressible implementation of CLSVOF dedicated to engine sprays [3]. This distribution, which follows closely a lognormal law, has been discretized using the two-size moment sectional method. The 3 sections and their characteristics are given in Table 2 . The bounds have been chosen in terms of droplet radius and allow to describe the polydisperse dynamics, with a different treatment of tracers, low inertia, and moderate inertia drops that is relevant to the present case. The two-size moment method preserves the mass flow rate ratios and the average $r_{30, k}$ of the lognormal distribution in each section. The tailored operator splitting here proves its capability to handle the two-way coupling between multiple sections and the gas, even in the near nozzle region.

The time evolution of the flow is presented in Fig. 13 and compares well to the behavior of Diesel sprays, with a rapid initial penetration followed by the slower development of the vapor-rich turbulent region. The behavior of the liquid phase is specifically presented in Fig. 14. It can be seen that the 


\begin{tabular}{l|ccc} 
Section number & 1 & 2 & 3 \\
\hline Upper bound $(\mu \mathrm{m})$ & 6 & 9.5 & 11.7 \\
Initial average radius $r_{30, k}(\mu \mathrm{m})$ & 3.88 & 7.37 & 11.68 \\
Mass flow rate ratio & $30.9 \%$ & $34.3 \%$ & $30.8 \%$ \\
Drag characteristic time $(\mu \mathrm{s})$ & 54 & 196 & 493
\end{tabular}

Table 2: Sectional discretization parameters as a model for the lognormal fit $\left(\mu_{L N}=1.9608\right.$ and $\left.\sigma_{L N}=0.4495\right)$ to the average size distribution obtained with a CLSVOF atomization DNS.

dense core behaves similarly for the three sections, while the sections respond with a different dynamics to the downstream vortices as soon as their mass loading is about or below $10 \%\left(\approx 70 \mathrm{~kg} / \mathrm{m}^{3}\right)$. The general aspects of entrainment and turbulence generation are retrieved as a result of the simultaneous coupling of all sections and gas through the splitting strategy. So the polydisperse approach emulates well the dense region.

The fields of fuel vapor and $\mathrm{CO}$, an intermediate reacting species, are given in Fig. 15. They extend beyond the liquid spray region and match the domain where Q-criterion indicates a high level of turbulence. Both these fields are corrugated and $\mathrm{CO}$ is significantly produced at the downstream end of the plume. Together with the equivalence ratio and temperature of Fig. 16, these quantities allow to analyze the mechanisms that lead to autoignition. The end of the plume has a large region where the temperature is above $800 \mathrm{~K}$ and the equivalence ratio $\varphi$ is close to 2 . At this temperature, high equivalence ratios are known to favor the so-called low-temperature reactions of the fuel so this rich region is a major candidate to investigate the path to autoignition $[65,54,36]$. The present strategy has therefore been able to finely describe the spray up to the point where it mixes as an ignitable turbulent flow.

\section{Conclusion and prospects}

A new transport scheme and coupling strategy have been developed for kinetic systems that are far from equilibrium (low pressure, low diffusion, low collisions) but strongly coupled to another phase. The approach is based on operator splitting of the coupled conservative system and on a forward semi-Lagrangian (FSL) algorithm for the transport of the disperse phase. The FSL transport scheme appears to be very promising for detailed computations of dense sprays, where gas-particle coupling is strong (two-way coupling) and has potential when particle-particle coupling is strong (collision, coalescence, or break-up). It has no noise and a natural balance of load as opposed to stochastic Lagrangian approaches. When using a linear projection, its implementation is simple and can be easily extended to spray methods with more moments. Conservation and realizability are guaranteed at the level of the projection algorithm. Its order and accuracy have been quantified on academic cases and its robustness and efficiency have been demonstrated on massively parallel computations of very dense to dense sprays with an initial mass loading of 35. The new strategy can be applied to any combination of Eulerian moment methods/closures. The demonstration of detailed simulation feasibility for the injection in Diesel engine conditions has been made using a polydisperse reacting spray model. These results are a first step before studying the autoignition sequence in more detail. Increasing the accuracy of the FSL strategy to second order is considered together with improving the modeling strategy in terms of inertial drop velocity closures and two-phase LES models.

\section{Acknowledgments}

Support by Sandia National Laboratories' Laboratory Directed Research and Development (LDRD) program is gratefully acknowledged. Sandia National Laboratories is a multi-program laboratory managed and operated by Sandia Corporation, a wholly owned subsidiary of Lockheed Martin Corporation, for the U.S. Department of Energy's National Nuclear Security Administration under contract DE-AC04-94AL85000. 


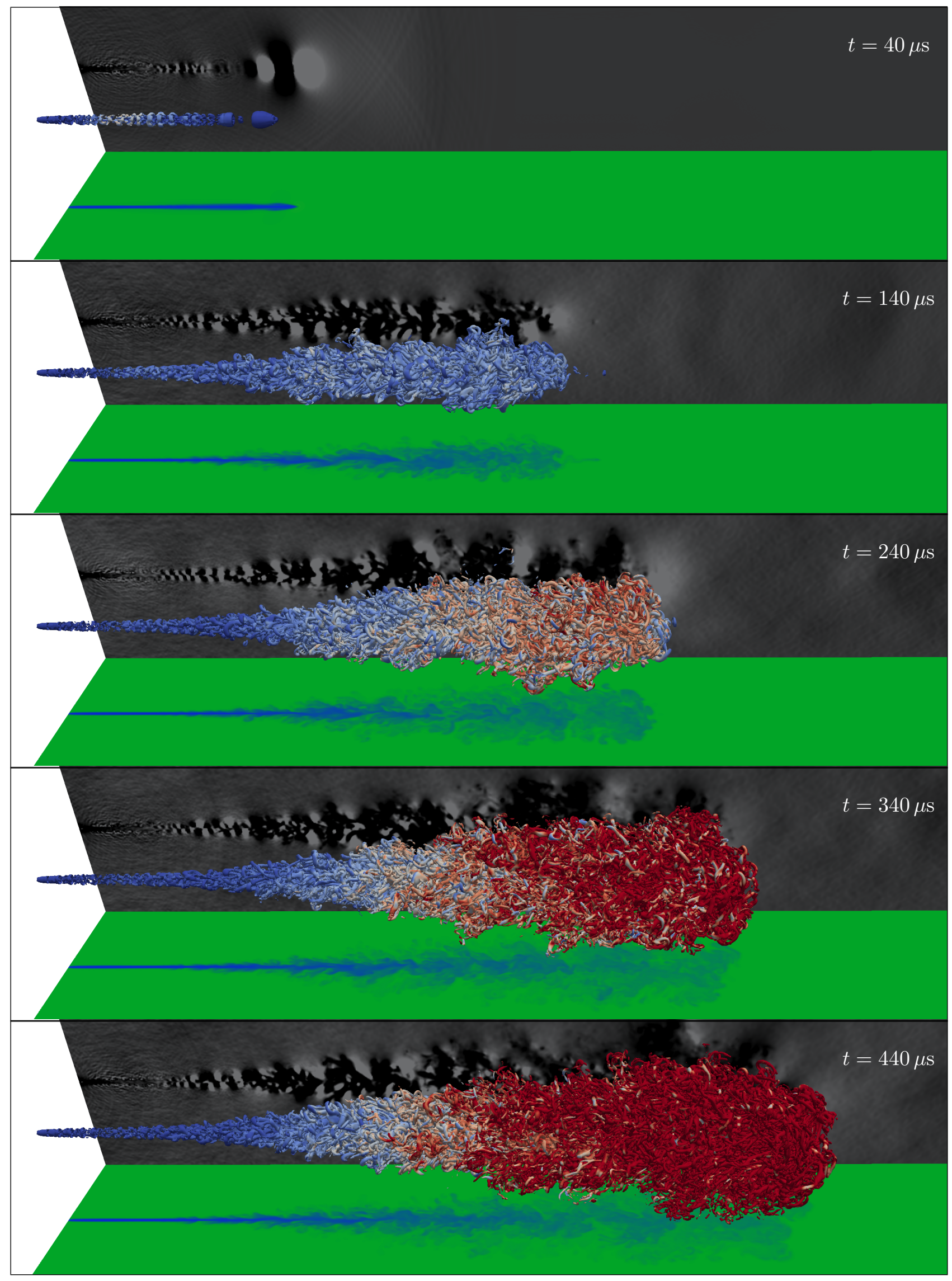

Figure 13: Injection sequence showing isocontour of Q-criterion colored by CO mass fraction (blue: $0 \%$; red: $0.1 \%$ ); Back plane: Gas pressure in the vertical center plane; Bottom plane: Gas temperature in the horizontal center plane. 

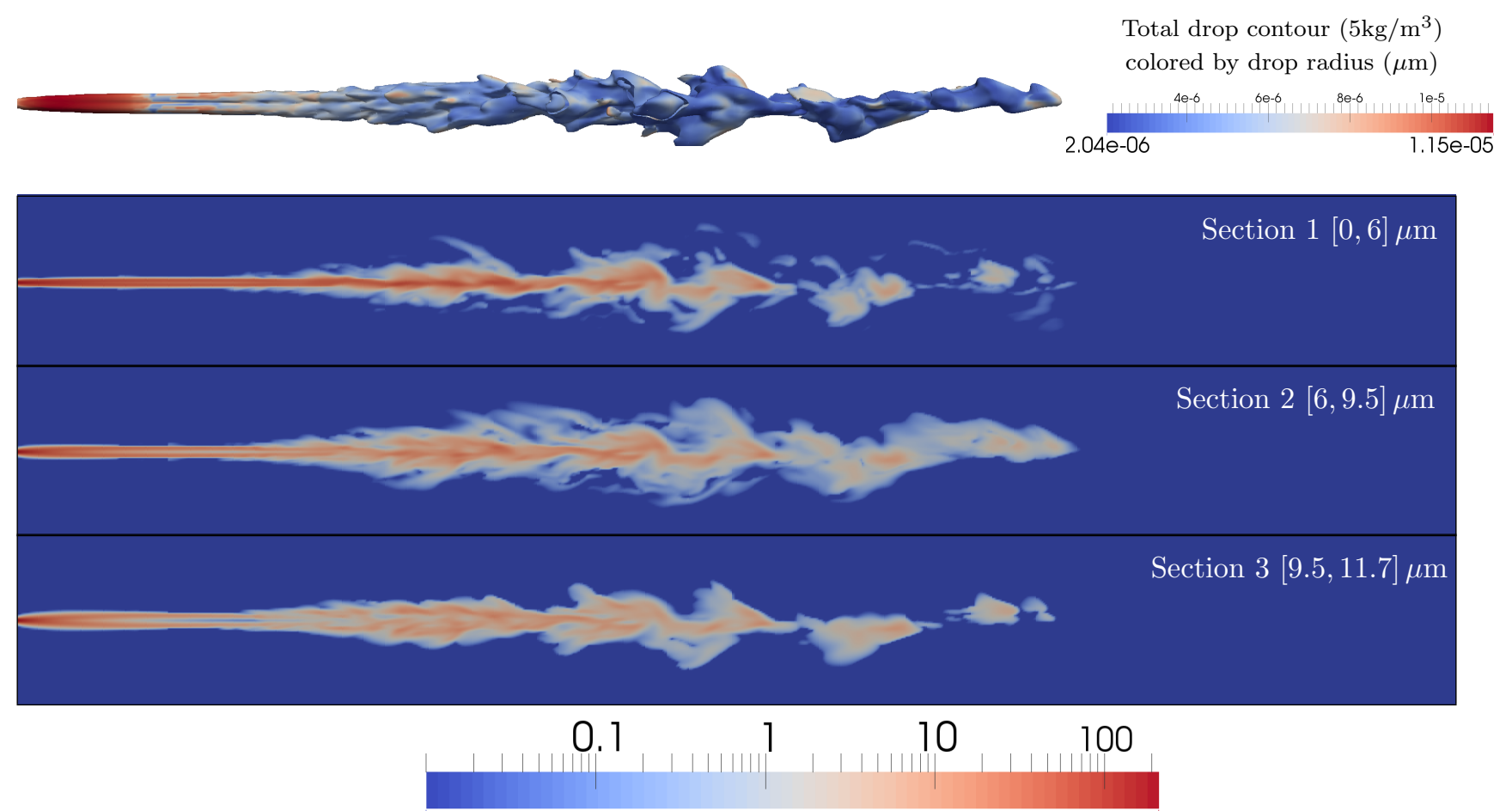

Figure 14: Spray mass density $\left(\mathrm{kg} / \mathrm{m}^{3}\right)$ in the reacting jet case at $t_{0}=400 \mu \mathrm{s}$.

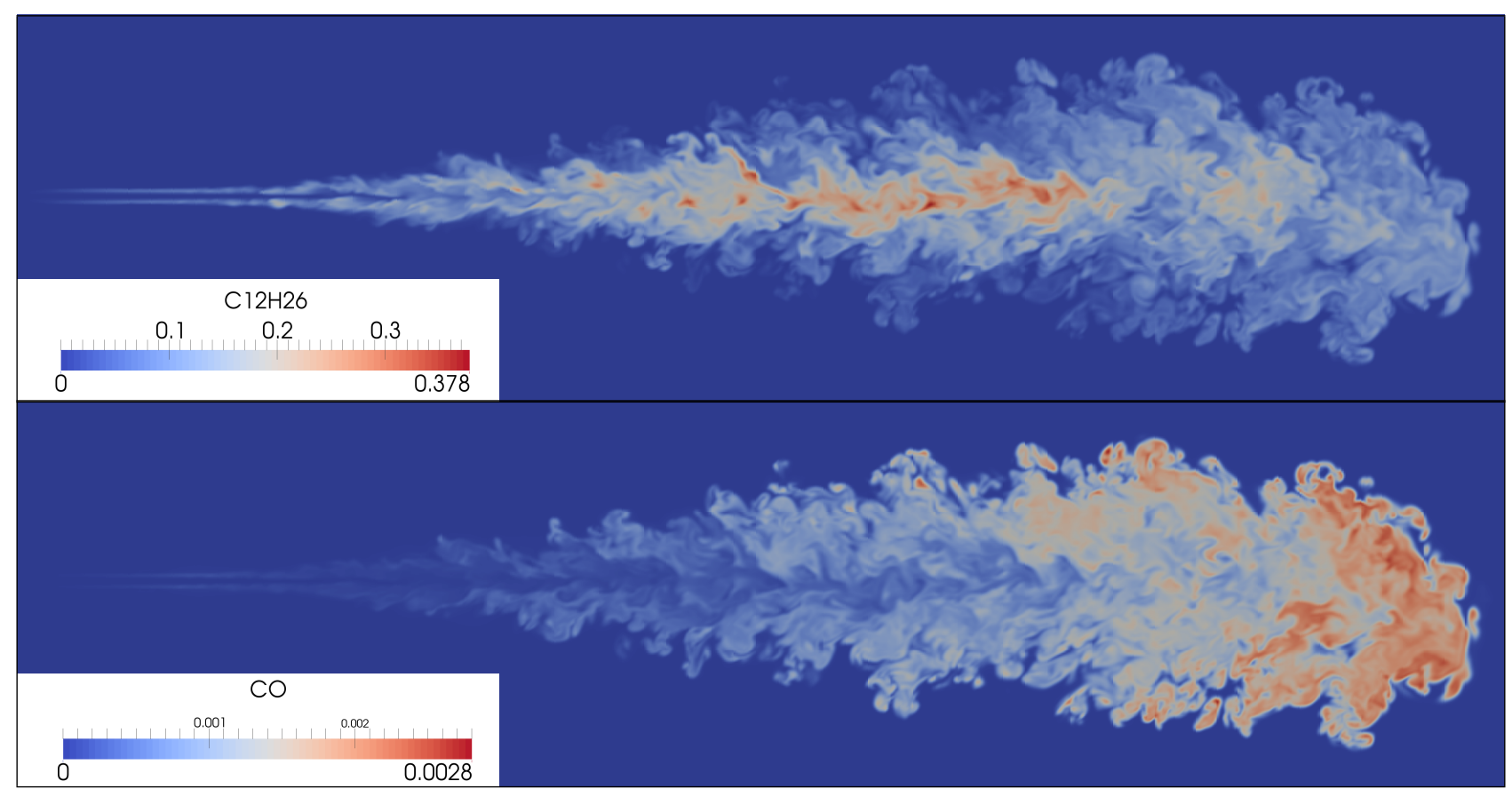

Figure 15: Fuel species in the reacting jet case at $t_{0}=400 \mu \mathrm{s} ;$ Top: n-dodecane; Bottom: carbon monoxide.

\section{Appendix A. Analytical study of 1D-cartesian advection}

When using the linear projection operation described in Section 2.3, the error of the FSL scheme can be studied analytically. Its analysis can be reduced to the advection of a single parcel (a Dirac $\delta$-distribution) thanks to linearity of this projection, since any spatial profile can be retrieved by superposition when no trajectory crossing occurs (linearity of transport). For the sake of simplicity, we hereafter assume that the grid is uniform and $1 \mathrm{D}$ with a characteristic length $\Delta x$. Also, the parcel 


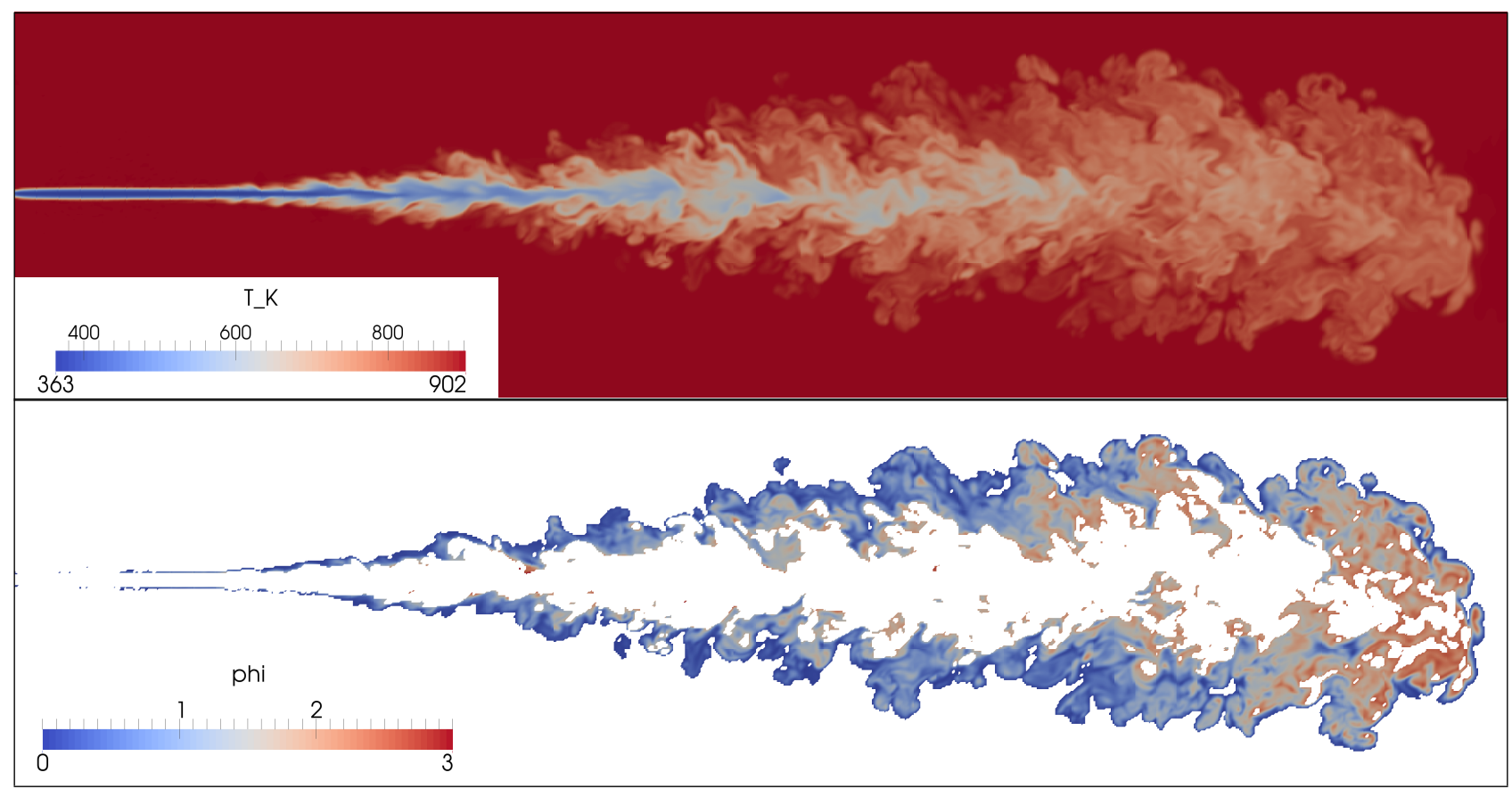

Figure 16: Thermochemistry fields in the reacting jet case at $t_{0}=400 \mu \mathrm{s}$; Top: gas temperature; Bottom: equivalence ratio $\varphi$ after temperature masking with $800 K<T_{g}<899 K$.

initially coincides with a cell center $x_{p}(0)=0$, and it moves at constant velocity $u$. Thus the CFL number is defined as $c=\frac{u \Delta t}{\Delta x}$. We describe the problem in dimensionless form so that $m_{p}=1$. After the first advection step $\left(t_{1}=\Delta t\right)$, the parcel is located at $x_{p}\left(t_{1}\right) / \Delta x=c$.

The projection step involves sharing the mass between the two neighbors, which are $x_{\lfloor c\rfloor}=\lfloor c\rfloor$ and $x_{\lceil c\rceil}=\lceil c\rceil$ if $\lfloor\cdot\rfloor$ and $\lceil\cdot\rceil$ are the floor and ceiling functions respectively. Note that the indices match the values due to non-dimensionalization and to the fact that $\lceil c\rceil=\lfloor c\rfloor+1$. The two neighbors respectively receive

$$
\begin{aligned}
& m_{\lfloor c\rfloor}=w_{\lfloor c\rfloor}\left(\eta_{\lfloor c\rfloor}\right)=1-\eta_{x}=1-\frac{x_{p}\left(t_{1}\right)-x_{\lfloor c\rfloor}}{x_{\lceil c\rceil}-x_{\lfloor c\rfloor}}=1-\{c\} \\
& m_{\lceil c\rceil}=w_{\lfloor c\rfloor+1}\left(\eta_{\lfloor c\rfloor+1}\right)=1-w_{\lfloor c\rfloor}\left(\eta_{\lfloor c\rfloor}\right)=\{c\},
\end{aligned}
$$

where the notation for the weights has been simplified from $w_{p i, x}$ to $w_{i}$ and where $\{\cdot\} \in[0,1]$ is the fractional part. This complies with all the weight requirements defined in Section 2.3. Meanwhile, the other grid points receive nothing; i.e., $m_{i}=0, i \neq\{\lfloor c\rfloor,\lceil c\rceil\}$ so that mass is conserved; i.e., $\sum m_{i}=(1-\{c\})+\{c\}=1$. The next transport step brings the two non-void parcels $p$ and $p^{\prime}$ to be located at

$$
\begin{array}{lc}
x_{p}\left(t_{2}\right)=x_{\lfloor c\rfloor}+c=\lfloor c\rfloor+c & \left(x_{\lfloor 2 c\rfloor}, x_{\lfloor 2 c\rfloor+1}\right) \\
x_{p^{\prime}}\left(t_{2}\right)=x_{\lceil c\rceil}+c=\lceil c\rceil+c
\end{array} \text { and projected on } \quad\left(x_{\lfloor 2 c\rfloor+1}, x_{\lfloor 2 c\rfloor+2}\right)
$$

so that the three grid points now involved with receiving mass are $x_{\lfloor 2 c-1\rfloor}, x_{\lfloor 2 c\rfloor}$, and $x_{\lfloor 2 c+1\rfloor}$ with

$$
\begin{aligned}
& m_{\lfloor 2 c-1\rfloor}=\{c\}^{2} \\
& m_{\lfloor 2 c\rfloor}=2\{c\}(1-\{c\}) \\
& m_{\lfloor 2 c+1\rfloor}=(1-\{c\})^{2},
\end{aligned}
$$

with the middle point receiving two contributions. This scheme exactly replicates Pascal's triangle such that the Eulerian masses can be deduced at each time step using a basic recursion. Because the problem is invariant by translation, we can now take the notation $m_{i}^{n}$ at time step $n$, where $i$ is a 
translated space index so that $m_{i}^{n}=0$ for $i$ outside of $\llbracket 0, n \rrbracket$. The fractional part of the CFL is now simply noted $c$, keeping in mind that the overall method is perfectly valid for $c>1$ but boils down to the result with $c^{\prime}=c-\lfloor c\rfloor \in[0,1[$ plus a translation of $n\lfloor c\rfloor$ grid points. We can finally solve the problem at every grid point $i$ and every time step $n$ using the binomial monoms

$$
m_{i}^{n}=\left(\begin{array}{c}
n \\
i
\end{array}\right) c^{n}(1-c)^{n-i} \quad \text { with } \quad\left(\begin{array}{c}
n \\
i
\end{array}\right)=\frac{n(n-1) \cdots(n-i+1)}{i(i-1) \cdots 1} .
$$

Mass conservation is retrieved using Newton's formula

$$
\sum_{i=0}^{n} m_{i}^{n}=(c+(1-c))^{n}=1 .
$$

The result after several advection-projection steps is given in Fig. A.17 in the case of CFL $=0.5$. The significant diffusion of mass to the neighbor cells is clearly visible.
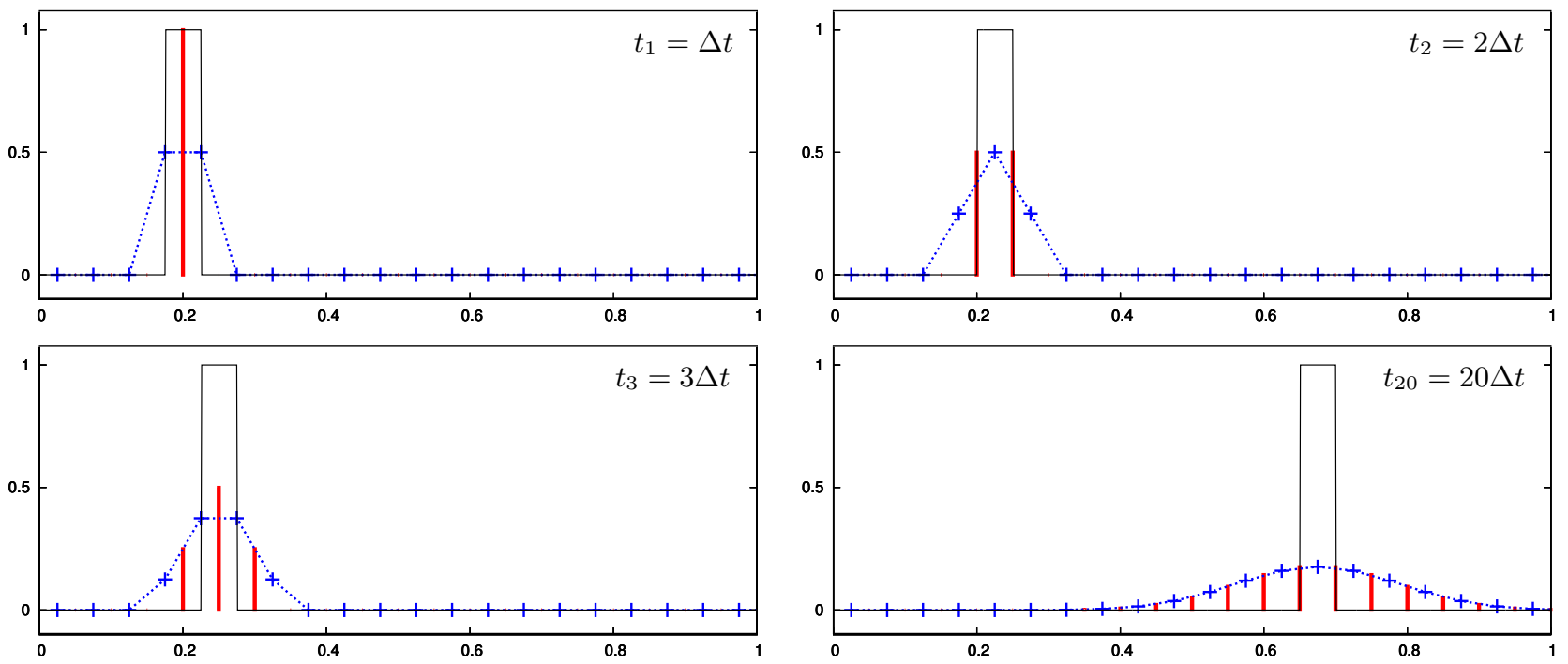

Figure A.17: Transport of the sharpest field possibly resolved (a discretized $\delta$-shock) using FSL-LP at CFL $c=0.5$; Solid line: Exact signal; Stick: Parcel after transport step; $\cdots+\cdots$ : Eulerian field after gather step.

Using the above analytical formula, we can exactly compute the numerical error of the method. For $n$ large enough, the binomial distribution behaves like the normal law with expectancy $\mathbb{E}\left(m_{i}^{n}\right)=n c$ and variance $\mathbb{V}\left(m_{i}^{n}\right)=n c(1-c)$. Moreover, this Gaussian distribution is the solution of an advectiondiffusion equation applied to the initial Dirac $\delta$-distribution. We refer to this equation as the so-called "modified equation" and it reads (in dimensioned form)

$$
\partial_{t} \rho+\partial_{x} \rho u_{\text {num }}=D_{\text {num }} \partial_{x x} \rho \quad \text { with } \quad u_{\text {num }}=\frac{c \Delta x}{\Delta t} \quad \text { and } \quad D_{\text {num }}=\frac{1}{2} \frac{\Delta x^{2}}{\Delta t}\{c\}(1-\{c\}) .
$$

By identification, we can state that the present FSL-LP scheme achieves an approximation of Eq. (A.1) that is of higher order compared to the approximation of the original system given by Eq. (3). Therefore the numerical errors can actually be quantified from the modified equation: the scheme is exact on group velocity $\left(u_{\text {num }}=u\right)$, it features diffusion $\left(D_{\text {num }} \geq 0\right)$, but it is non-dispersive since no additional odd spatial derivatives are present. The modified equation also shows that the Lagrangian transport coupled to a projection operation renders the exact solution for $c \in \mathbb{N}$ (yielding $D_{\text {num }}=0$ ) while diffusion error is maximum for $\{c\}=1 / 2$. Numerical diffusion is expected to smear the sharpest profiles just as physical diffusion would. This effect is illustrated in Fig. A.18 on the advection of a signal presenting a discontinuity and a continuous span of space frequencies. After 25 time steps, the highest frequencies appear to be significantly smeared out but the scheme is strictly non-oscillatory 


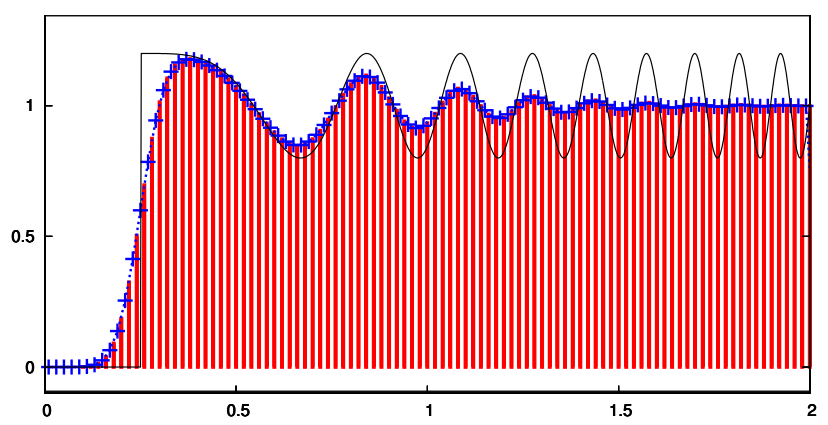

Figure A.18: Transport of a multi-scale signal using the FSL-LP scheme at CFL $c=0.5$ after 25 time steps; Solid line: Exact signal; Stick: Parcel after transport step; $\cdots+\cdots$ : Eulerian field after gather step.

and positivity-preserving.

Remark: If we consider weights $w_{x}\left(\eta_{x}\right)$ that are non-linear in $\eta_{x}$ and where the non-linear function still enforces the above properties, especially $w_{i}\left(1-\eta_{x}\right)=1-w_{i}\left(\eta_{x}\right)$, the same developments can be made by substituting $w_{i}(\{c\})$ to $\{c\}$ so that we get the instantaneous positions

$$
m_{i}^{n}=\left(\begin{array}{c}
n \\
i
\end{array}\right) w_{i}(\{c\})^{n}\left(1-w_{i}(\{c\})\right)^{n-i}
$$

It is obvious that mass is again conserved thanks to the symmetry property. However, we now have $\mathbb{E}\left(m_{i}^{n}\right)=n w_{i}(\{c\})$ and variance $\mathbb{V}\left(m_{i}^{n}\right)=n w_{i}(\{c\})\left(1-w_{i}(\{c\})\right)$. This is interesting from the point of view of diffusion since we can get $D_{\text {num }}^{\prime}$ arbitrarily close to 0 for any $c$. Unfortunately the advection velocity $u_{\text {num }}=\frac{w_{i}(\{c\}) \Delta x}{\Delta t}=\frac{w_{i}(\{c\})}{c} u$ is directly affected by the non-linear process, being altered by a factor $\frac{w_{i}(\{c\})}{c}$. To summarize, a non-linear weighting repartition leads to either decreased phase velocity and decreased diffusion or increased phase velocity and increased diffusion. Thus diffusion error has been traded for dispersion error. The linear weights are the only possible ones to perform a non-dispersive projection when purely relying on the geometry to compute the weights.

\section{References}

[1] A. A. Amsden, P. J. O'Rourke, and T. D. Butler. Kiva II, a computer program for chemically reactive flows with sprays. Technical Report LA-11560-MS, Los Alamos National Laboratory, Los Alamos, New Mexico, 1989.

[2] S.V. Apte, K. Mahesh, P. Moin, and J.C. Oefelein. Large-eddy simulation of swirling particle-laden flows in a coaxial-jet combustor. Int. J. Multiph. Flows, 29(8):1311-1331, 2003.

[3] M. Arienti and M. Sussman. A High-Fidelity Study of High-Pressure Diesel Injection. SAE Tech. Paper 01-1853, 2015.

[4] S. Balachandar and J.K. Eaton. Turbulent dispersed multiphase flow. Ann. Rev. of Fluid Mec., 42:111-133, 2010.

[5] M. Bardi, R. Payri, L.-M. Malbec, G. Bruneaux, L. M. Pickett, J. Manin, T. Bazyn, and C. Genzale. Engine Combustion Network: comparison of spray development, vaporization, and combustion in different combustion vessels. Atom. Spr., 22(10), 2012.

[6] C. Bardos, F. Golse, and D. Levermore. Fluid dynamic limits of kinetic equations. I. Formal derivations. Journal of Statistical Physics, 63, 1991.

[7] A. Bernard-Champmartin, J.-P. Braeunig, C. Fochesato, and T. Goudon. A semi-Lagrangian approach for dilute non-collisional fluid-particle flows. Communications in Computational Physics, 19(3):801-840, 2016.

[8] P. Bertrand, G. Baumann, and M.R. Feix. Frequency shift of non linear electron plasma oscillation. Physics Letters A, 29(9):489-490, 1969.

[9] N. Besse. Convergence of a semi-Lagrangian scheme for the one-dimensional Vlasov-Poisson system. SIAM Journal on Numerical Analysis, 42(1):350-382, 2004.

[10] N. Besse, F. Berthelin, Y. Brenier, and P. Bertrand. The multi-water-bag equations for collisionless kinetic modeling. Kinet. Relat. Models, 2(1):39-80, 2009.

[11] G. A. Bird. Molecular gas dynamics and the direct simulation of gas flows, volume 42. Oxford Science Publications, 1994.

[12] C.K. Birdsall and A.B. Langdon. Plasma physics via computer simulation. CRC Press, 2004.

[13] M. Boileau, G. Staffelbach, B. Cuenot, T. Poinsot, and C. Bérat. LES of an ignition sequence in a gas turbine engine. Comb. Flame, 154(1):2-22, 2008. 
[14] P. Bosler, L. Wang, C. Jablonowski, and R. Krasny. A Lagrangian particle/panel method for the barotropic vorticity equations on a rotating sphere. Fluid Dynamics Research, 46(3):031406, 2014.

[15] F. Bouchut. On zero pressure gas dynamics. In Advances in kinetic theory and computing, pages 171-190. World Sci. Publishing, River Edge, NJ, 1994.

[16] F. Bouchut, S. Jin, and X. Li. Numerical approximations of pressureless and isothermal gas dynamics. SIAM J. Num. Anal., 41:135-158, 2003.

[17] J. Capecelatro and O. Desjardins. Mass loading effects on turbulence modulation by particle clustering in dilute and moderately dilute channel flows. Journal of Fluids Engineering, 137(11):111102, 2015.

[18] J.A. Carrillo and F. Vecil. Nonoscillatory interpolation methods applied to Vlasov-based models. SIAM Journal on Scientific Computing, 29(3):1179-1206, 2007.

[19] C. Chalons, M. Massot, and A. Vié. On the Eulerian Large Eddy Simulation of disperse phase flows: an asymptotic preserving scheme for small Stokes number flows. Multiscale Modeling ES Simulation, 13(1):291-315, 2015.

[20] C.-Z. Cheng and G. Knorr. The integration of the Vlasov equation in configuration space. J. Comp. Phys., 22(3):330-351, 1976.

[21] B. Cockburn, G. E. Karniadikis, and C. W. Shu. Discontinuous Galerkin Methods - Theory, Computation, and Applications, volume 11 of Lecture Notes in Computer Science and Engineering. Springer Verlag, New York, 2000.

[22] C.J. Cotter, J. Frank, and S. Reich. The remapped particle-mesh semi-Lagrangian advection scheme. Quarterly Journal of the Royal Meteorological Society, 133(622):251-260, 2007.

[23] G.-H. Cottet, J.-M. Etancelin, F. Pérignon, and C. Picard. High order semi-Lagrangian particle methods for transport equations: numerical analysis and implementation issues. ESAIM. Mathematical Modelling and Numerical Analysis, 48(4):1029, 2014.

[24] G.H. Cottet and P.-A. Raviart. On particle-in-cell methods for the Vlasov-Poisson equations. Transport Theory and Statistical Physics, 15(1-2):1-31, 1986.

[25] R. Courant, E. Isaacson, and M. Rees. On the solution of nonlinear hyperbolic differential equations by finite differences. Communications on Pure and Applied Mathematics, 5(3):243-255, 1952.

[26] N. Crouseilles, M. Mehrenberger, and E. Sonnendrücker. Conservative semi-Lagrangian schemes for Vlasov equations. J. Comp. Phys., 229(6):1927-1953, 2010.

[27] N. Crouseilles, T. Respaud, and E. Sonnendrücker. A forward semi-lagrangian method for the numerical solution of the Vlasov equation. Computer Physics Communications, 180(10):1730-1745, 2009.

[28] C. Crowe, M. Sharma, and D. Stock. The particle-source-in cell (PSI-CELL) model for gas-droplet flows. J. Fluids Eng., 99(2):325-332, 1977.

[29] S. de Chaisemartin. Polydisperse evaporating spray turbulent dispersion: Eulerian model and numerical simulation. $\mathrm{PhD}$ thesis, Ecole Centrale Paris, 2009.

[30] J. Denavit. Numerical simulation of plasmas with periodic smoothing in phase space. J. Comp. Phys., 9(1):75-98, 1972.

[31] D.C. DePackh. The water-bag model of a sheet electron beamy. International Journal of Electronics, 13(5):417-424, 1962.

[32] S. Descombes and M. Massot. Operator splitting for nonlinear reaction-diffusion systems with an entropic structure: singular perturbation and order reduction. Num. Math., 97(4):667-698, 2004.

[33] F. Doisneau. Eulerian modeling and simulation of polydisperse moderately dense coalescing spray flows with nanometric-to-inertial droplets: application to Solid Rocket Motors. PhD thesis, Ecole Centrale Paris, 2013.

[34] F. Doisneau, M. Arienti, and J. C. Oefelein. Space-filtered kinetic theory for the LES of dense sprays. In $13^{\text {th }}$ Triennial International Conference on Liquid Atomization and Spray Systems, Tainan, Taiwan, 23-27 August 2015.

[35] F. Doisneau, M. Arienti, and J. C. Oefelein. Study of the momentum coupling between liquid fuel and ambient gas during injection using a dense spray formulation. In $9^{\text {th }}$ U.S. National Combustion Meeting, Cincinnati, USA, 17-20 May 2015.

[36] F. Doisneau, M. Arienti, and J. C. Oefelein. On Multi-Fluid models for spray-resolved LES of reacting jets. Proc. Comb. Inst., pages 1-9, 2017. Accepted.

[37] F. Doisneau, J. Dupays, A. Murrone, F. Laurent, and M. Massot. Eulerian VS Lagrangian simulation of unsteady two-way coupled coalescing two-phase flows in solid propellant combustion. C. R. Mec., 341:44-54, 2013. Special issue "Combustion, spray and flow dynamics for aerospace propulsion".

[38] F. Doisneau, F. Laurent, A. Murrone, J. Dupays, and M. Massot. Eulerian Multi-Fluid models for the simulation of dynamics and coalescence of particles in solid propellant combustion. J. Comp. Phys., 234:230-262, 2013.

[39] F. Doisneau, A. Sibra, J. Dupays, A. Murrone, F. Laurent, and M. Massot. Numerical strategy for unsteady two-way coupled polydisperse sprays: Application to solid-rocket instabilities. J. Prop. Power, 30(3):727-747, 2014.

[40] J. K. Dukowicz. A particle-fluid numerical model for liquid sprays. J. Comp. Phys., 35(2):229-253, 1980.

[41] O. Emre, R.O. Fox, M. Massot, S. De Chaisemartin, S. Jay, and F. Laurent. Towards eulerian modeling of a polydisperse evaporating spray under realistic internal-combustion-engine conditions. Flow. Turb. Comb., 93(4):689$722,2014$.

[42] O. Emre, D. Kah, S. Jay, Q.-H. Tran, A. Velghe, S. De Chaisemartin, R.O. Fox, F. Laurent, and M. Massot. Eulerian moment methods for automotive sprays. Atomization and Sprays, 25(3), 2015.

[43] M. Falcone and R. Ferretti. Semi-Lagrangian approximation schemes for linear and Hamilton-Jacobi equations. Cambridge University Press, 2014. 
[44] P. Fede, O. Simonin, and A. Ingram. 3D numerical simulation of a lab-scale pressurized dense fluidized bed focussing on the effect of the particle-particle restitution coefficient and particle-wall boundary conditions. Chemical Engineering Science, 142:215-235, 2016.

[45] J. H. Ferziger and H. G. Kaper. Mathematical Theory of transport processes in gases. North-Holland, Amsterdam, 1972.

[46] F. Filbet and E. Sonnendrücker. Comparison of eulerian Vlasov solvers. Computer Physics Communications, 150(3):247-266, 2003.

[47] F. Filbet, E. Sonnendrücker, and P. Bertrand. Conservative numerical schemes for the Vlasov equation. J. Comp. Phys., 172(1):166-187, 2001.

[48] R. O. Fox. On multiphase turbulence models for collisional fluid-particle flows. J. Fluid Mech., 742:368-424, 2014.

[49] L. Fréret, O. Thomine, F. Laurent, J. Reveillon, and M. Massot. Direct Numerical Simulation of polydisperse evaporating sprays in 3D jet configuration using Euler-Euler and Euler-Lagrange formalisms. In Proc. Summer Program 2012, pages 345-354, Center for Turbulence Research, Stanford University, 2012.

[50] M. Garcia. Development and validation of the Euler-Lagrange formulation on a parallel and unstructured solver for large-eddy simulation. PhD thesis, Université Toulouse III, 2009. available online at http://ethesis.inptoulouse.fr/archive/00000715/.

[51] S.K. Godunov. A difference method for numerical calculation of discontinuous solutions of the equations of hydrodynamics. Matematicheskii Sbornik, 47(3):271-306, 1959.

[52] V. Grandgirard, M. Brunetti, P. Bertrand, N. Besse, X. Garbet, P. Ghendrih, G. Manfredi, Y. Sarazin, O. Sauter, and E. Sonnendrücker. A drift-kinetic semi-Lagrangian 4D code for ion turbulence simulation. J. Comp. Phys., 217(2):395-423, 2006.

[53] M. Gros, P. Bertrand, and M.R. Feix. Connection between hydrodynamic, water bag and Vlasov models. Plasma Physics, 20(10):1075, 1978.

[54] L. Hakim, G. Lacaze, M. Khalil, H.N. Najm, and J.C. Oefelein. Modeling Auto-Ignition Transients in Reacting Diesel Jets. J. Eng. Gas Turb. Power, 138(11):112806, 2016.

[55] A. Hamiaz, M. Mehrenberger, A. Back, and P. Navaro. Guiding center simulations on curvilinear grids. ESAIM. Proceedings and Surveys, 53, 2016.

[56] A. Hamiaz, M. Mehrenberger, H. Sellama, and E. Sonnendrücker. The semi-Lagrangian method on curvilinear grids. 2015. Available on hal.archives-ouvertes.fr.

[57] J. J. Hylkema. Modélisation cinétique et simulation numérique d'un brouillard dense de gouttelettes. Application aux propulseurs à poudre. PhD thesis, École Nationale Supérieure de l'Aéronautique et de l'Espace, 1999.

[58] A. Irannejad, A. Banaeizadeh, and F. Jaberi. Large eddy simulation of turbulent spray combustion. Comb. Flame, 162(2):431-450, 2015.

[59] M. Jemison, M. Sussman, and M. Arienti. Compressible, multiphase semi-implicit method with moment of fluid interface representation. J. Comp. Phys., 279:182-217, 2014.

[60] W.P. Jones, A.J. Marquis, and K. Vogiatzaki. Large-eddy simulation of spray combustion in a gas turbine combustor. Combustion and Flame, 161(1):222-239, 2014.

[61] D. Kah, O. Emre, Q.H. Tran, S. Chaisemartin, S. Jay, F. Laurent, and M. Massot. High order moment method for polydisperse evaporating sprays with mesh movement: Application to internal combustion engines. Int. J. Multiph. Flows, 71:38-65, 2015.

[62] A. Kitagawa, Y. Murai, and F. Yamamoto. Two-way coupling of Eulerian-Lagrangian model for dispersed multiphase flows using filtering functions. Int. J. Multiph. Flows, 27(12):2129-2153, 2001.

[63] C. Klingenberg and K. Waagan. Relaxation solvers for ideal MHD equations-a review. Acta Mathematica Scientia, 30(2):621-632, 2010.

[64] T. N. Krishnamurti. Numerical integration of primitive equations by a quasi-Lagrangian advective scheme. Journal of applied Meteorology, 1(4):508-521, 1962.

[65] G. Lacaze, A. Misdariis, A. Ruiz, and J.C. Oefelein. Analysis of high-pressure Diesel fuel injection processes using LES with real-fluid thermodynamics and transport. Proc. Comb. Inst., 2015.

[66] F. Laurent and M. Massot. Multi-fluid modeling of laminar poly-dispersed spray flames: origin, assumptions and comparison of the sectional and sampling methods. Comb. Th. Model., 5:537-572, 2001.

[67] F. Laurent, M. Massot, and P. Villedieu. Eulerian Multi-Fluid modeling for the numerical simulation of coalescence in polydisperse dense liquid sprays. J. Comp. Phys., 194:505-543, 2004.

[68] F. Laurent, A. Sibra, and F. Doisneau. Two-size moment multi-fluid model: a robust and high-fidelity description of polydisperse moderately dense evaporating sprays. Commun. Comput. Phys., 20:902-943, 2016.

[69] R. L. Liboff. Kinetic theory: classical, quantum, and relativistic descriptions. Springer Science \& Business Media, 2003.

[70] A. Magni and G.H. Cottet. Accurate, non-oscillatory, remeshing schemes for particle methods. J. Comp. Phys., 231(1):152-172, 2012.

[71] L. Martinez, A. Benkenida, and B. Cuenot. A model for the injection boundary conditions in the context of 3D simulation of Diesel spray: methodology and validation. Fuel, 89:219-228, 2010.

[72] E. Masi, O. Simonin, E. Riber, P. Sierra, and L.Y.M. Gicquel. Development of an algebraic-closure-based moment method for unsteady Eulerian simulations of particle-laden turbulent flows in very dilute regime. Int. J. Multiph. Flows, 58:257-278, 2014. 
[73] R. D. Nair, J. S. Scroggs, and F. H. M. Semazzi. A forward-trajectory global semi-Lagrangian transport scheme. J. Comp. Phys., 190(1):275-294, 2003.

[74] J. C. Oefelein. Large Eddy Simulation of turbulent combustion processes in propulsion and power systems. Prog. Aerosp. Sc., 42(1):2-37, 2006.

[75] P. J. O'Rourke. Collective drop effects on vaporizing liquid sprays. PhD thesis, Los Alamos National Laboratory 87545, University of Princeton, 1981.

[76] R. Paoli, T. Poinsot, and K. Shariff. Testing semi-Lagrangian schemes for two-phase flow applications. In Proceeding of the Summer Program. Stanford CTR, 2006.

[77] Y. Pei, E. R Hawkes, M. Bolla, S. Kook, G. M Goldin, Y. Yang, S.B. Pope, and S. Som. An analysis of the structure of an n-dodecane spray flame using TPDF modelling. Comb. Flame, 168:420-435, 2016.

[78] L.M. Portela and R.V. Oliemans. Eulerian-Lagrangian DNS/LES of particle-turbulence interactions in wallbounded flows. International Journal for Numerical Methods in Fluids, 43(9):1045-1065, 2003.

[79] T. Respaud and E. Sonnendrücker. Analysis of a new class of forward semi-Lagrangian schemes for the 1D VlasovPoisson equations. Numerische Mathematik, 118(2):329-366, 2011.

[80] A. Robert. A semi-Lagrangian and semi-implicit numerical integration scheme for the primitive meteorological equations. J. Meteor. Soc. Jap., 60(1):319-325, 1982.

[81] M. Sabat, A. Larat, A. Vié, and M. Massot. On the development of high order realizable schemes for the Eulerian simulation of disperse phase flows : a convex-state preserving Discontinuous Galerkin method. J. Comp. Multiphase Flows, 6(3):247-270, 2014.

[82] C.W. Shu and S. Osher. Efficient implementation of essentially non-oscillatory shock-capturing schemes. J. Comp. Phys., 77(2):439-471, 1988.

[83] A. Sibra, J. Dupays, F. Laurent, and M. Massot. A new Eulerian Multi-Fluid model for bi-component polydisperse sprays: an essential approach to evaluate the impact of aluminum combustion on Solid Rocket Motor instabilities. In $49^{\text {th }}$ AIAA/ASME/SAE/ASEE Joint Propulsion Conference, San Jose, U.S.A., 14-17 July 2013.

[84] P. K. Smolarkiewicz and J. A. Pudykiewicz. A class of semi-Lagrangian approximations for fluids. J. Atm. Sc., 49(22):2082-2096, 1992.

[85] E. Sonnendrücker, J. Roche, P. Bertrand, and A. Ghizzo. The semi-Lagrangian method for the numerical resolution of the Vlasov equation. J. Comp. Phys., 149(2):201-220, 1999.

[86] A. Staniforth and J. Côté. Semi-Lagrangian integration schemes for atmospheric models-a review. Monthly Weather Review, 119(9):2206-2223, 1991.

[87] S. Tachibana, K. Saito, T. Yamamoto, M. Makida, T. Kitano, and R. Kurose. Experimental and numerical investigation of thermo-acoustic instability in a liquid-fuel aero-engine combustor at elevated pressure: Validity of large-eddy simulation of spray combustion. Combustion and Flame, 162(6):2621-2637, 2015.

[88] J. Tillou, J.-B. Michel, C. Angelberger, and D. Veynante. Assessing LES models based on tabulated chemistry for the simulation of diesel spray combustion. Comb. Flame, 161(2):525-540, 2014.

[89] B. Van Leer. Toward the ultimate conservation difference scheme V. a second-order sequel to Godunov's method. J. Comp. Phys., 32(1):101-136, 1979.

[90] I. Verma, E. Bish, M. Kuntz, E. Meeks, K. Puduppakkam, C. Naik, and L. Liang. CFD modeling of spark ignited gasoline engines-Part 2: Modeling the engine in Direct Injection mode along with spray validation. Technical report, SAE Technical Paper, 2016.

[91] A. Vié, F. Doisneau, and M. Massot. On the Anisotropic Gaussian velocity closure for inertial-particle laden flows. Commun. Comput. Phys., 17:1-46, 2015.

[92] K. Waagan, C. Federrath, and C. Klingenberg. A robust numerical scheme for highly compressible magnetohydrodynamics: Nonlinear stability, implementation and tests. J. Comp. Phys., 230(9):3331-3351, 2011.

[93] A. Wehrfritz, O. Kaario, V. Vuorinen, and B. Somers. Large Eddy Simulation of n-dodecane spray flames using Flamelet Generated Manifolds. Comb. Flame, 167:113-131, 2016.

[94] A Wiin-Nielsen. On the application of trajectory methods in numerical forecasting. Tellus, 11(2):180-196, 1959.

[95] F. A. Williams. Spray combustion and atomization. Phys. Fluids, 1:541-545, 1958.

[96] R. Zamansky, F. Coletti, M. Massot, and A. Mani. Radiation induces turbulence in particle-laden fluids. Phys. Fluids, 26(7):071701, 2014.

[97] Y. B. Zel'dovich. Gravitational instability : an approximate theory for large density perturbations. Astronomy and Astrophysics, 5:84-89, 1970. 